\title{
Stability for nonautonomous linear differential systems with infinite delay
}

\author{
TERESA FARIA \\ Departamento de Matemática and CMAF-CIO, Faculdade de Ciências, Universidade de Lisboa \\ Campo Grande, 1749-016 Lisboa, Portugal \\ Email: teresa.faria@fc.ul.pt
}

To the memory of Professor Russell A. Johnson

\begin{abstract}
We study the stability of general $n$-dimensional nonautonomous linear differential equations with infinite delays. Delay independent criteria, as well as criteria depending on the size of some finite delays are established. In the first situation, the effect of the delays is dominated by nondelayed diagonal negative feedback terms, and sufficient conditions for both the asymptotic and the exponential asymptotic stability of the system are given. In the second case, the stability depends on the size of some bounded diagonal delays and coefficients, although terms with unbounded delay may co-exist. Our results encompass DDEs with discrete and distributed delays, and enhance some recent achievements in the literature.
\end{abstract}

Keywords: linear delay differential equations, infinite delay, exponential stability, asymptotic stability.

2010 Mathematics Subject Classification: 34K06, 34K20, 34K25.

\section{Introduction}

In this paper, the focus is to investigate the asymptotic and exponential stabilities of a general nonautonomous linear system of delay differential equations (DDEs) with infinite delay,

$$
x^{\prime}(t)=\mathcal{L}(t) x_{t}, \quad t \in I,
$$

where $I=\left[t_{0}, \infty\right)$ for some $t_{0} \in \mathbb{R}, \mathcal{L}(t)$ is in $L\left(\mathcal{C}, \mathbb{R}^{n}\right)$, the usual space of bounded linear operators from $\mathcal{C}$ to $\mathbb{R}^{n}$ equipped with the operator norm, and $\mathcal{C}$ is an adequate Banach space of continuous functions defined on $(-\infty, 0]$ with values in $\mathbb{R}^{n}$. As usual, $x_{t}$ denotes the entire past history of the system up to time $t$, or, in other words, $x_{t}(s)=x(t+s)$ for $s \leq 0$. For simplicity, here one assumes that $(t, \phi) \mapsto \mathcal{L}(t) \phi$ is continuous, although one could consider the more general framework of $t \mapsto \mathcal{L}(t) \phi$ a Borel measurable function for each $\phi$, with $\|\mathcal{L}(t)\|$ bounded on $I$ by a function $m(t)$ in $L_{\text {loc }}^{1}(I ; \mathbb{R})$. See [22, Chapter 4] for more details.

The stability of autonomous and nonautonomous linear DDEs has been the subject of intensive studies. Even for ordinary differential equations (ODEs), the nonautonomous situation is not easy to address in its generality, see e.g. important contributions by Coppel [7], Johnson and Sell [24], Sacker and Sell [31]. On the other hand, the introduction of large delays in differential equations may lead to oscillations, loss of stability of equilibria, and existence of unbounded solutions. For linear DDEs, delay independent results for stability as well as criteria depending on the size of the delays have been established, for both scalar and multi-dimensional equations. The literature on this 
subject is very vast: here we only refer to some monographs [16, 20, 26] and a few selected papers [1, 9, 11, 19, 23, 25, 32, 33, 34].

For the last few years, there has been a renewed interest in the analysis of stability of nonautonomous linear DDEs, and several methods and tools have been proposed, see e.g. [2, 4, 5, 14, 15, [17, 18, 21, 28, 29] and references therein. The main goal of this paper is to obtain new explicit sufficient conditions for the asymptotic and exponential asymptotic stability of a general linear system (1.1), which improve and generalize some criteria in recent literature. We emphasize that here we consider very general linear DDEs with possible unbounded delays, both discrete and distributed, while typically most authors impose the delays to be finite or discrete, or both. Moreover, the a priori boundedness of all the coefficients will not be required. Two types of criteria will be obtained, depending on whether system (1.1) possesses diagonal terms without delay which dominate the effect of the delayed terms, or not. The latter case is not often treated in the literature, although there have been some recent interesting developments in this area [2, 4, 5, 14, 33], following different approaches: 3/2-stability conditions, Lyapounov functionals, theory of monotone systems, asymptotic equivalence to linear ODEs, etc.

The method employed here is based on an auxiliary simple result, which states that, under some algebraic conditions and without imposing the boundedness of coefficients and delays, the norm along solutions is nonincreasing. Special care is however required to deal with the infinite delay. Our techniques are very different from others proposed in the literature, though the results presented in this paper were inspired by some previous works, which prompted us to search for either sharper or more embracing criteria. Some of our concrete purposes are described below.

In 13, Faria and Oliveira gave sharp conditions for the exponential asymptotic stability of autonomous linear systems with finite delay and dominating instantaneous negative feedbacks. The analysis in [13] was further pursued in [10], for the case of infinite delay. Ngoc and Cao [28] considered a linear system, again with dominating diagonal terms without delay, of the form $x^{\prime}(t)=-D(t) x(t)+$ $\mathcal{L}(t) x_{t}$, where $\mathcal{L}(t)$ has the form $\mathcal{L}(t) \phi=\int_{-\infty}^{0} B(t, s) \phi(s) d s$ and $D(t), B(t, \cdot)$ are $n \times n$ matrices of continuous functions, but assumed that $D(t), B(t, \cdot)$ are bounded by some autonomous matrices. One of the goals of the present paper is to remove this constraint. Recently, Hatvani [21] and Györi and Horváth [17] achieved sharper results for the asymptotic stability of scalar differential equations $x^{\prime}(t)=-d(t) x(t)+\beta(t) x(t-\tau(t))$ and inequalities $x^{\prime}(t) \leq-d(t) x(t)+\beta(t) x(t-\tau(t))$, respectively, without the a priori requirement of having bounded coefficients. We shall show that some of the results in [17, 18, 21, 28] are a simple consequence of the stability criteria established here for $n$ dimensional linear DDEs. On the other hand, there are several recent works where explicit conditions for the exponential asymptotic stability of linear DDEs depending on the size of delays were found, see Berezansky and Braverman [2, 3] for the scalar case and Berezansky et al. 4, 5] for $n$-dimensional systems, as well as references therein. However, in [2, 4] only the situation of time-varying bounded and discrete delays was considered - two constraints removed in this work. In any case, for criteria depending on the delays, clearly constraints on the size of some diagonal delays must be imposed.

We observe that not only the asymptotic stability of general linear equations (1.1) is important per se, but also that it has relevant consequences in the study of the global dynamics of nonautonomous DDEs

$$
x^{\prime}(t)=\mathcal{L}(t) x_{t}+f\left(t, x_{t}\right),
$$

where $f$ is smooth on some open subset of $\mathbb{R} \times \mathcal{C}$. This system can be seen as a perturbation of (1.1), and the stability or instability of its linearization at e.g. 0 (if $f(t, 0)=0, D_{2} f(t, 0)=0$ for all $t$ ) is 
a key ingredient to further analyze the large-time behavior of solutions, in terms of local or global asymptotic stability, existence of oscillatory solutions, and many other features. In the autonomous case $x^{\prime}(t)=\mathcal{L} x_{t}+f\left(x_{t}\right)$, where $f(0)=0, f^{\prime}(0)=0$, the well-known principle of linearized stability is valid for equations with infinite delay [8]. The nonautonomous situation is certainly more difficult to analyze, but a crucial idea is to use the variation of constant formula and the stability properties of the linearized system, possibly coupled with additional properties of the nonlinear perturbation $f$, such as monotonicity or boundedness, to further derive sufficient conditions for the stability, persistence and permanence of (1.2). This methodology was used for instance in [12], where the authors studied the asymptotic behavior of solutions for a family of nonlinear DDEs obtained as perturbations of an ODE, given by $x^{\prime}(t)=A(t) x(t)+f\left(t, x_{t}\right)$, with $A(t)$ an $n \times n$ matrix of continuous functions such that the ODE $x^{\prime}(t)=A(t) x(t)$ is exponentially stable, and $f$ of the form $f(t, \phi)=\left(f_{1}\left(t, \phi_{1}\right), \ldots, f_{n}\left(t, \phi_{n}\right)\right)$ for $t \geq 0$ and $\phi=\left(\phi_{1}, \ldots, \phi_{n}\right)$ with $(t, \phi) \in \operatorname{dom} f$. In fact, a main motivation for this work was to first address the stability of linear DDEs (1.1), in order to extend the results in [12] to some classes of nonautonomous linear DDEs (1.2) with infinite delay.

This paper is organized as follows. In Section 2, a suitable phase space $\mathcal{C}$ to treat DDEs with infinite delay is chosen, and some notation introduced. In Section 3, we consider a linear DDE with dominant nondelayed terms, start with some auxiliary results and then establish delay-independent sufficient conditions for both its exponential and asymptotic stabilities. In the latter case, some further restrictions on the general form of (1.1) are imposed. In Section 4, we study the stability of (1.1) without assuming the existence and dominance of diagonal instantaneous negative feedback terms; nevertheless it turns out that the size of the diagonal coefficients and finite delays will be decisive to derive our stability criteria, although unbounded delays may co-exist. Through Sections 3 and 4, we compare our results with some achievements in recent literature. In the last section, some illustrative examples are presented.

\section{Phase space and notation}

In this preliminary section, we recall an abstract framework to deal with DDEs with infinite delay. In view of the unbounded delays, the phase space $\mathcal{C}$ should satisfy some fundamental axioms which guarantee that it is 'admissible', so that the classical results of existence, uniqueness, continuation for the future, and continuous dependence of solutions on the initial data are valid - a subject well establish in the literature. A convenient choice of $\mathcal{C}$ is set below, however other spaces are possible.

Consider a weight function $g$ satisfying the following properties:

(g) $g:(-\infty, 0] \rightarrow[1, \infty)$ is a nonincreasing continuous function such that $g(0)=1, \lim _{s \rightarrow-\infty} g(s)=\infty$ and $\lim _{u \rightarrow 0^{-}} \frac{g(s+u)}{g(s)}=1$ uniformly on $(-\infty, 0]$.

For each $n \in \mathbb{N}$, define the Banach space

$$
C_{g}^{0}=C_{g}^{0}\left(\mathbb{R}^{n}\right):=\left\{\phi \in C\left((-\infty, 0] ; \mathbb{R}^{n}\right): \lim _{t \rightarrow-\infty} \frac{|\phi(s)|}{g(s)}=0\right\},
$$

with the norm

$$
\|\phi\|_{g}=\sup _{s \leq 0} \frac{|\phi(s)|}{g(s)}
$$


and $|\cdot|$ any chosen norm in $\mathbb{R}^{n}$. This space is an admissible Banach phase space in the sense that it satisfies the required axioms (A), (B) and (C2) of [22]. For instance, for any $\gamma>0$ the function $g(s)=\mathrm{e}^{-\gamma s}, s \leq 0$, satisfies the properties in (g); for such $g$, the notations $C_{\gamma}^{0}:=C_{\mathrm{e}^{-\gamma}}^{0}$. and $\|\phi\|_{\gamma}:=\sup _{s \leq 0} \mathrm{e}^{\gamma s}|\phi(s)|$ are used. Alternatively, the space $C_{\gamma}=C_{\gamma}\left(\mathbb{R}^{n}\right):=\left\{\phi \in C\left((-\infty, 0] ; \mathbb{R}^{n}\right)\right.$ : $\lim _{t \rightarrow-\infty} \mathrm{e}^{\gamma s}|\phi(s)|$ exists $\}$ with the same norm $\|\phi\|_{\gamma}$ is often considered in the literature.

In $\mathcal{C}=C_{g}^{0}$, an $n$-dimensional DDE with infinite delay is written in abstract form as

$$
x^{\prime}(t)=f\left(t, x_{t}\right),
$$

where $f: D \subset \mathbb{R} \times \mathcal{C} \rightarrow \mathbb{R}^{n}$ is continuous (or satisfies the Caratheodory conditions), and, as usual, the entire past of unkown solutions in the phase space $\mathcal{C}$ are denoted by $x_{t}: x_{t}(s)=x(t+s), s \leq 0$.

The space $C_{g}^{0}$ as well as $C_{\gamma}$ are always fading memory spaces, which provides some further important properties for solutions of (2.1) [27]. In what concerns linear autonomous equations $x^{\prime}(t)=L x_{t}$, with $L \in L\left(\mathcal{C}, \mathbb{R}^{n}\right)$, it is well known that, if $\mathcal{C}$ is a fading memory space, then the zero solution is asymptotically stable if and only if all the roots of the characteristic equation have negative real parts. See [22, 27] for definitions, results and more properties.

Clearly, the case of systems $x^{\prime}(t)=f\left(t, x_{t}\right)$ with finite delay is included in the present setting. In fact, for DDEs with finite delay $\tau \geq 0$, take a weight function $g:(-\infty, 0] \rightarrow[1, \infty)$ such that $g(s) \equiv 1$ on $[-\tau, 0]$ and $(\mathrm{g})$ holds. Thus, the space $C\left([-\tau, 0] ; \mathbb{R}^{n}\right)$ with the usual sup norm $\|\cdot\|_{\infty}$ can be seen as a closed subspace of $C_{g}^{0}$ with the norm $\|\cdot\|_{g}$.

The dual $\left(C_{g}^{0}(\mathbb{R})\right)^{\prime}$ of $C_{g}^{0}(\mathbb{R})$ is identified with the space $M_{g}((-\infty, 0] ; \mathbb{R})$ of Borel measures $\mu$ : $(-\infty, 0] \rightarrow \mathbb{R}$, in the sense that each bounded linear functional $T: C_{g}^{0}(\mathbb{R}) \rightarrow \mathbb{R}$ is represented by a real Borel measure $\mu:(-\infty, 0] \rightarrow \mathbb{R}$,

$$
T(\psi)=\int_{-\infty}^{0} \psi(s) d \mu(s), \quad t \geq 0, \psi \in C_{g}(\mathbb{R}),
$$

with operator norm $\|T\|=\operatorname{Var}_{(-\infty, 0]}(g \mu):=\int_{-\infty}^{0} g(s) d|\mu|(s)<\infty$ [30]. Thus, an operator $L \in$ $L\left(\mathcal{C}, \mathbb{R}^{n}\right)$ is identified with an element $\eta=\left[\eta_{i j}\right]_{n \times n}$ in the space $M_{g}\left((-\infty, 0] ; \mathbb{R}^{n \times n}\right)$ of $n \times n$ matrixvalued Borel measures on $(-\infty, 0], \eta_{i j} \in M_{g}((-\infty, 0] ; \mathbb{R})$, in such a way that $L \phi=\int_{-\infty}^{0}[d \eta(s)] \phi(s)$, with norm $\|L\|=\|\eta\|_{g}:=\int_{-\infty}^{0} g(s) d|\eta|(s)<\infty$, where $|\eta|(s)$ is the total variation measure of $\eta(s)$.

We now set some notation and terminology. As mentioned, the phase space is a priori fixed as $\mathcal{C}=C_{g}^{0}$, for some weight function $g$ satisfying (g). A vector $c$ in $\mathbb{R}^{n}$ is said to be positive if all its components are positive, and we write $c>0$. Analogously, we define nonnegative vectors $c$, and positive and nonnegative functions $\phi \in \mathcal{C}$, with notation $c \geq 0, \phi>0, \phi \geq 0$, respectively. A vector $c$ in $\mathbb{R}^{n}$ is identified in $\mathcal{C}$ with the constant function $\phi(s)=c$ for $s \leq 0$. For $c \in \mathbb{R}^{n}$, it is understood that $c_{i}$ means the $i$ th-component of $c$, for $1 \leq i \leq n$. Analogously, $f_{i}$ is the $i$ th-component of a function $f$ with values in $\mathbb{R}^{n}$.

Unless otherwise stated, we suppose that $\mathbb{R}^{n}$ is equipped with the supremum norm, $|x|=|x|_{\infty}=$ $\max _{1 \leq i \leq n}\left|x_{i}\right|$, for $x=\left(x_{1}, \ldots, x_{n}\right) \in \mathbb{R}^{n}$. If there is no possibility of misinterpretation, the norm $\|\cdot\|_{g}$ in $\mathcal{C}$ will be simply denoted by $\|\cdot\|$. For a positive vector $v=\left(v_{1}, \ldots, v_{n}\right)$ we denote by $v^{-1}$ the vector $v^{-1}=\left(v_{1}^{-1}, \ldots, v_{n}^{-1}\right)$; we shall also consider norms $|\cdot|_{v}$ defined by $|x|_{v}=\max _{1 \leq i \leq n}\left(v_{i}\left|x_{i}\right|\right)$ for $x=$ $\left(x_{1}, \ldots, x_{n}\right) \in \mathbb{R}^{n}$ and the corresponding norms in $\mathcal{C}$, given by $\|\varphi\|_{v}=\|\varphi\|_{g, v}=\sup _{s \leq 0} g(s)^{-1}|\varphi(s)|_{v}$. Hereafter, we use $\mathbf{1}=(1, \ldots, 1)$. 
Solutions of linear systems (1.1) with initial conditions $x_{\sigma}=\phi(\sigma \in I, \phi \in \mathcal{C})$, i.e., $x(\sigma+s)=$ $\phi(s), s \leq 0$, are defined for all $t \geq \sigma[22]$; they are denoted by $x(t, \sigma, \phi)$ in $\mathbb{R}^{n}$, or $x_{t}(\sigma, \phi)$ in $\mathcal{C}$. In what follows, let $I=\mathbb{R}^{+}:=[0, \infty)$, but any other choice of $I=\left[t_{0}, \infty\right)$ is possible. Here, for simplicity, the concepts of asymptotic and exponential stability always refer to stability on some interval $[\alpha, \infty) \subset \mathbb{R}^{+}$. Note also that, for linear systems, the asymptotic stability is equivalent to the stability and global attractivity of $x=0$. To be more precise, we will use the definitions below.

Definition 2.1. The linear system (1.1) is said to be stable on $[\alpha, \infty) \subset \mathbb{R}^{+}$if for any $\varepsilon>0$ and $\sigma \geq \alpha$ there is $\delta=\delta(\varepsilon, \sigma)>0$ such that $\left\|x_{t}(\sigma, \phi)\right\|<\varepsilon$ for all $t \geq \sigma$, whenever $\|\phi\|<\delta$. System (1.1) is asymptotically stable if $x=0$ is asymptotically stable on some interval $[\alpha, \infty) \subset \mathbb{R}^{+}$; in other words, (1.1) is stable on $[\alpha, \infty)$ and $\lim _{t \rightarrow \infty} x(t, \sigma, \phi)=0$ for all $\sigma \geq 0, \phi \in \mathcal{C} ; x=0$ is exponentially asymptotically stable if all solutions of (1.1) tend to zero exponentially at infinity, uniformly on some interval $[\alpha, \infty) \subset \mathbb{R}^{+}$; i.e., there exist $\alpha, K, \beta>0$ such that $\left\|x_{t}(\sigma, \phi)\right\| \leq K \mathrm{e}^{-\beta(t-\sigma)}\|\phi\|$ for all $t \geq \sigma \geq \alpha$ and $\phi \in \mathcal{C}$.

\section{Linear systems with instantaneous diagonal dominance}

In this section, we deal with linear DDEs for which the effect of the delays is dominated by nondelayed diagonal negative feedback terms. In order to analyze the absolute stability, i.e., to set stability conditions which do not dependent on the delays, we separate nondelayed from delayed terms in $\mathcal{L}(t)$, so that $\mathcal{L}(t)$ has the form $\mathcal{L}(t) \phi=-D(t) \phi(0)+L(t) \phi$, and write the nonautonomous linear system (1.1) as

$$
x^{\prime}(t)=-D(t) x(t)+L(t) x_{t}, \quad t \in I,
$$

where $D(t)=\left[d_{i j}(t)\right]$ is an $n \times n$ matrix of functions on $I=\mathbb{R}^{+}$, and $L: I \rightarrow L\left(\mathcal{C}, \mathbb{R}^{n}\right)$. Terms with time-dependent discrete delays as well as distributed delays are all incorporated in $L(t) x_{t}$.

For $L(t)$ as in (3.1), let $\eta(t)=\left[\eta_{i j}(t, \cdot)\right]_{n \times n} \in M_{g}\left((-\infty, 0] ; \mathbb{R}^{n \times n}\right)$ be such that

$$
L(t) \phi=\int_{-\infty}^{0}\left[d_{s} \eta(t, s)\right] \phi(s) \quad \text { for } \quad \phi \in \mathcal{C},
$$

with norm given by $\|L(t)\|=\|\eta(t)\|_{g}:=\int_{-\infty}^{0} g(s) d|\eta|(t, \cdot)(s)<\infty$. Consider the components $L(t)=\left(L_{1}(t), \ldots, L_{n}(t)\right)$, and write $L_{i}(t) \phi=\sum_{j=1}^{n} L_{i j}(t) \phi_{j}$ for $t \geq 0, \phi=\left(\phi_{1}, \ldots, \phi_{n}\right) \in \mathcal{C}$, where each linear functional $L_{i j}(t): C_{g}(\mathbb{R}) \rightarrow \mathbb{R}$ is identified with $\eta_{i j}(t, \cdot) \in M_{g}((-\infty, 0] ; \mathbb{R})$.

With (1.1) written in this form, one may suppose that the operators $L_{i j}(t)$ are non-atomic at zero, i.e., $\eta_{i j}\left(t, 0^{-}\right)=\eta_{i j}(t, 0)$ for $t \geq 0$ (cf. [20, Chapter 6]), this restriction however will not have any influence in the writing of our results.

Define the $n \times n$ matrix-valued functions

$$
\begin{aligned}
& D(t)=\left[d_{i j}(t)\right], \quad \widehat{D}(t)=\left[\hat{d}_{i j}(t)\right] \text { where } \hat{d}_{i j}(t)=\left\{\begin{array}{rl}
d_{i i}(t) & \text { if } i=j \\
-\left|d_{i j}(t)\right| & \text { if } i \neq j
\end{array},\right. \\
& A(t)=\left[\left\|L_{i j}(t)\right\|\right] \quad \text { and } \quad M(t)=\widehat{D}(t)-A(t), \quad \text { for } \quad t \in[0, \infty) .
\end{aligned}
$$

For (3.1), in the sequel we assume the general hypotheses:

(H1) the functions $d_{i j}:[0, \infty) \rightarrow \mathbb{R}, L_{i j}:[0, \infty) \rightarrow L\left(C_{g}^{0}(\mathbb{R}), \mathbb{R}\right)$ are continuous, for $i, j=1, \ldots, n$; 
(H2) there exist a vector $v>0$ and $T \geq 0$ such that $M(t) v \geq 0$ for $t \geq T$.

Remark 3.1. To simplify the exposition, here the regularity in (H1) is imposed. In fact, as mentioned in the Introduction, instead of continuous functions one could consider the more general framework of $d_{i j}(t)$ in $L_{\text {loc }}^{1}\left(\mathbb{R}^{+} ; \mathbb{R}\right)$ and $t \mapsto L(t) \phi$ Borel measurable for each $\phi$, with $\|L(t)\|$ bounded on $\mathbb{R}^{+}$by a function $m(t)$ in $L_{\text {loc }}^{1}\left(\mathbb{R}^{+} ; \mathbb{R}\right)$. We stress that the proofs of our results do not depend on the continuity of the coefficients, as the reader can easily verify. Thus, it is important to notice that in particular they are generalized in a straightforward way to linear DDEs with impulses, where coefficients and delays are piecewise continuous functions.

A dominance of the diagonal instantaneous terms in (3.1) is expressed by condition (H2). Note also that (H2) implies that $d_{i}(t):=d_{i i}(t) \geq 0$ for all $t \geq 0, i \in\{1, \ldots, n\}$, with the equality $d_{i}(t)=0$ if and only if all the coefficients of the $i$ th-lines of $D(t)$ and $A(t)$ are zero.

Remark 3.2. Consider (3.1) under the general assumption (H1), and suppose that (H2) is satisfied. Set $a_{i j}(t):=\left\|L_{i j}(t)\right\|$. Rescaling the variables by $\bar{x}_{i}(t)=v_{i}^{-1} x_{i}(t)(1 \leq i \leq n)$, where $v=$ $\left(v_{1}, \ldots, v_{n}\right)>0$ is a vector as in (H2), we obtain a new linear DDE $\bar{x}^{\prime}(t)=-\bar{D}(t) \bar{x}(t)+\bar{L}(t) \bar{x}_{t}$, where the corresponding matrices $\bar{D}(t)=\left[\bar{d}_{i j}(t)\right]$ and $\bar{A}(t)=\left[\bar{a}_{i j}(t)\right]$ have entries $\bar{d}_{i j}(t)=v_{i}^{-1} d_{i j}(t) v_{j}$ and $\bar{a}_{i j}(t)=v_{i}^{-1} a_{i j}(t) v_{j}$. In this way, and after dropping the bars for simplicity, we may consider a system (3.1) for which (H2) is valid with $v=\mathbf{1}:=(1, \ldots, 1)$. This scaling of $\mathbb{R}^{n}$ also transforms the norm $|x|_{v^{-1}}=\max _{1 \leq i \leq n} v_{i}^{-1}\left|x_{i}\right|$ into the norm $|x|=\max _{1 \leq i \leq n}\left|x_{i}\right|$. Throughout this paper, without loss of generality and whenever it is convenient, if condition (H2) (or a stronger version of (H2), see (H4), (H5) below) is satisfied, we shall assume that it holds with the vector $v=\mathbf{1}$.

We start with some preliminary lemmas. The auxiliary result below, although elementary, plays a crucial role in our stability criteria.

Lemma 3.1. If assumptions (H1), (H2) are satisfied, the solutions of (1.1) satisfy $\left|x\left(t, t_{0}, \phi\right)\right|_{v^{-1}} \leq$ $\|\phi\|_{g, v^{-1}}$ for $t \geq t_{0} \geq T, \phi \in \mathcal{C}$, where $T, v$ are as in (H2). In particular, (1.1) is (uniformly) stable on $[T, \infty)$.

Proof. As described above, after rescaling the variables by $\bar{x}_{i}(t)=v_{i}^{-1} x_{i}(t)(1 \leq i \leq n)$ we may assume $(\mathrm{H} 2)$ with $v=\mathbf{1}:=(1, \ldots, 1)$, which in turn implies $d_{i}(t) \geq \sum_{j \neq i}\left|d_{i j}(t)\right|+\sum_{j}\left\|L_{i j}(t)\right\|$ for all $i$ and $t \geq T$, and $|x|_{v^{-1}}=\max _{1 \leq i \leq n}\left|x_{i}\right|$.

Fix $\phi \in \mathcal{C}, t_{0} \geq T$, and consider the solution $x(t)=x\left(t, t_{0}, \phi\right)$ of (1.1). We claim that $|x(t)| \leq$ $\left\|x_{t_{0}}\right\|_{g}$ for $t$ on each interval $\left[t_{0}, t_{0}+a\right](a>0)$.

Define $J=\left[t_{0}, t_{0}+a\right]$. For the sake of contradiction, suppose that $\max _{t \in J}|x(t)|>\left\|x_{t_{0}}\right\|_{g}$. Denote $u_{j}=\max _{t \in J}\left|x_{j}(t)\right|$ for $1 \leq j \leq n$, and take $i \in\{1, \ldots, n\}$ and $t_{1} \in\left(t_{0}, t_{0}+a\right]$ such that $u_{i}=\max _{j} u_{j}=\left|x_{i}\left(t_{1}\right)\right|=\left|x\left(t_{1}\right)\right|$. For $s \leq 0$ and $t \in J$, we get

$$
\frac{|x(t+s)|}{g(s)} \leq \frac{|x(t+s)|}{g\left(t-t_{0}+s\right)} \leq\left\|x_{t_{0}}\right\|_{g}<\left|x_{i}\left(t_{1}\right)\right| \quad \text { if } \quad s+t \leq t_{0}
$$

and

$$
\frac{|x(t+s)|}{g(s)} \leq|x(t+s)| \leq\left|x_{i}\left(t_{1}\right)\right| \quad \text { if } \quad t_{0} \leq s+t
$$


hence $\left\|x_{t}\right\|_{g} \leq u_{i}$ for $t \in J$. Now, we suppose that $x_{i}\left(t_{1}\right)>0$; the case $x_{i}\left(t_{1}\right)<0$ is analogous. For $d_{i}(t)=d_{i i}(t)$, from $(\mathrm{H} 2)$ we obtain

$$
x_{i}^{\prime}(t)+d_{i}(t) x_{i}(t) \leq \sum_{j \neq i}\left|d_{i j}(t)\left\|x_{j}(t) \mid+\sum_{j}\right\| L_{i j}(t)\|\| x_{j, t} \|_{g} \leq d_{i}(t) u_{i}, \quad t \in J,\right.
$$

thus

$$
x_{i}(t) \leq x_{i}\left(t_{0}\right) \mathrm{e}^{-\int_{t_{0}}^{t} d_{i}(s) d s}+u_{i}\left(1-\mathrm{e}^{-\int_{t_{0}}^{t} d_{i}(s) d s}\right), \quad t \in J
$$

For $t=t_{1}$, we derive $x_{i}\left(t_{0}\right)-u_{i} \geq 0$, which contradicts the assumption $u_{i}>\left\|x_{t_{0}}\right\|_{g}$.

Lemma 3.2. Consider (3.1) and assume (H1). In addition, suppose that there exists a measurable, locally integrable function $e: \mathbb{R} \rightarrow \mathbb{R}^{+}$, such that the following conditions are satisfied:

(i) the operators

$$
\tilde{L}_{i j}(t)(\psi):=L_{i j}(t)\left(\psi^{t, e}\right),
$$

where $\psi^{t, e}(s):=\mathrm{e}^{\int_{t+s}^{t} e(u) d u} \psi(s)$, for $s \leq 0, t \gg 1$ and $\psi \in C_{g}^{0}(\mathbb{R})$, are well-defined, $i, j=1, \ldots, n$;

(ii) for some vector $v>0,[\widehat{D}(t)-\tilde{A}(t)-e(t) I] v \geq 0$ for $t \gg 1$, where $\tilde{A}(t)=\left[\left\|\tilde{L}_{i j}(t)\right\|\right]$ and $I$ is the $n \times n$ identity matrix;

(iii) $\int_{0}^{\infty} e(t) d t=\infty$.

Then (3.1) is asymptotically stable. In other words, (3.1) is stable (on some interval $[\alpha, \infty)$ ) and all its solutions satisfy $\lim _{t \rightarrow \infty} x(t)=0$. Moreover, if conditions (i), (ii) are satisfied with $e(t) \equiv \delta>0$, then (3.1) is exponentially asymptotically stable.

Proof. With $e(t) \geq 0$, (ii) implies (H2), thus (3.1) is stable. Set $\tilde{a}_{i j}(t)=\left\|\tilde{L}_{i j}(t)\right\|$, for $\tilde{L}_{i j}(t)$ in (3.3) . Without loss of generality, take $v=\mathbf{1}$ in (ii) (see Remark 3.2) and $T \geq 0$, so that

$$
d_{i}(t)-\sum_{j \neq i}\left|d_{i j}(t)\right|-\sum_{j} \tilde{a}_{i j}(t)-e(t) \geq 0, \quad t \geq T, i=1, \ldots, n .
$$

Effect the change of variables $y(t)=\mathrm{e}^{E(t)} x(t)$, where $E(t)=\int_{0}^{t} e(u) d u$. The linear DDE (3.1) is transformed into

$$
y_{i}^{\prime}(t)=-\left(d_{i}(t)-e(t)\right) y_{i}(t)-\sum_{j \neq i} d_{i j}(t) y_{j}(t)+\sum_{j} \tilde{L}_{i j}(t)\left(y_{j, t}\right), i=1, \ldots, n, t \geq 0 .
$$

In virtue of (3.4), this transformed system satisfies (H2). Let $x(t)=x\left(t, t_{0}, \phi\right)$ be a solution of the original equation. From Lemma 3.1, it follows that $|y(t)|=\left|y\left(t, t_{0}, \mathrm{e}^{E(\cdot)} \phi\right)\right| \leq\left\|\mathrm{e}^{E(\cdot)} \phi\right\|_{g} \leq\|\phi\|_{g}$ for $t \geq t_{0} \geq T$, thus $\left|x\left(t, t_{0}, \phi\right)\right| \leq \mathrm{e}^{-E(t)}\|\phi\|_{g}$ for all $t \geq t_{0} \geq T$ and $\phi \in \mathcal{C}$. As $\mathrm{e}^{-E(t)} \rightarrow 0$ as $t \rightarrow \infty$, then $\lim _{t \rightarrow \infty} x(t)=0$ for all solutions of (3.1). With $E(t)=\delta t$ for some $\delta>0$, we obtain $\left|x\left(t, t_{0}, \phi\right)\right| \leq \mathrm{e}^{-\delta t}\|\phi\|_{g}$ for $t \geq t_{0} \geq T$ and $\phi \in \mathcal{C}$, and (3.1) is exponentially asymptotically stable.

For future reference, we mention that a closer look to the proof of Lemma 3.2 shows that the requirement of having $e(t)$ a nonnegative function is only used to derive that (3.1) is stable, since with $e(t) \geq 0$ condition (ii) in Lemma 3.2 implies (H2), and that $\left\|\mathrm{e}^{E(\cdot)} \phi\right\|_{g} \leq\|\phi\|_{g}$. For the case of finite delays, condition (i) above always holds and, to derive only the global attractivity of the zero solution, $e(t)$ need not be nonnegative. 
In what follows, we shall assume some additional requirements on $D(t), L(t)$, in order to have conditions (i)-(iii) of Lemma 3.2 satisfied. For simplicity, we write

$$
L_{i j}(t)(\psi)=a_{i j}(t) \int_{-\infty}^{0} \psi(s) d_{s} \nu_{i j}(t, s), \quad t \geq 0, \psi \in C_{g}(\mathbb{R})
$$

where the functions $\nu_{i j}(t, s)$ are measurable in $(t, s) \in \mathbb{R}^{+} \times(-\infty, 0]$, continuous in $t \geq 0$, leftcontinuous in $s \in(-\infty, 0)$, with $g(s) \nu_{i j}(t, s)$ of bounded variation in $s \in(-\infty, 0]$ and $\nu_{i j}(t, s)$ normalized (relative to the norm $\|\cdot\|_{g}$ in $C_{g}(\mathbb{R})$ ), so that

$$
a_{i j}(t):=\left\|L_{i j}(t)\right\| \quad \text { and } \quad \int_{-\infty}^{0} g(s) d_{s}\left|\nu_{i j}\right|(t, s)=1
$$

Therefore, nonautonomous linear systems (3.1) are written in a more descriptive way as

$$
x_{i}^{\prime}(t)=-\sum_{j=1}^{n} d_{i j}(t) x_{j}(t)+\sum_{j=1}^{n} a_{i j}(t) \int_{-\infty}^{0} x_{j}(t+s) d_{s} \nu_{i j}(t, s), i=1, \ldots, n, t \geq 0 .
$$

Note that each component $L_{i}(t) x_{t}=\sum_{j=1}^{n} L_{i j}(t) x_{j, t}$ may contain terms with several time-dependent discrete delays, as well as distributed delays. In particular, each $L_{i j}(t) x_{j, t}$ may be of the form

$$
L_{i j}(t) x_{j, t}=-\sum_{k=1}^{p} d_{i j}^{k}(t) x_{j}\left(t-\tau_{i j}^{k}(t)\right)+\alpha_{i j}(t) \int_{-\infty}^{0} x_{j}(t+s) d_{s} \nu_{i j}(t, s),
$$

or

$$
L_{i j}(t) x_{j, t}=-\sum_{k=1}^{p} d_{i j}^{k}(t) \int_{-\tau_{i j}^{k}(t)}^{0} x_{j}(t+s) d_{s} \xi_{i j}^{k}(t, s)+\alpha_{i j}(t) \int_{-\infty}^{0} x_{j}(t+s) d_{s} \nu_{i j}(t, s),
$$

with $s \mapsto \xi_{i j}^{k}(t, s), \nu_{i j}(t, s)$ normalized so that $\int_{-\tau_{i j}^{k}(t)}^{0} g(s) d_{s}\left|\xi_{i j}^{k}\right|(t, s)=\int_{-\infty}^{0} g(s) d_{s}\left|\nu_{i j}\right|(t, s)=1$ for all $i, j, k$. In this case, $\left\|L_{i j}(t)\right\| \leq \sum_{k=1}^{p}\left|d_{i j}^{k}(t)\right|+\left|\alpha_{i j}(t)\right|$. So far, our approach does not require any special treatment of the terms with delays $\tau_{i j}^{k}(t)$ in (3.7) or (3.8) - although the last results of this section concern systems with $L_{i j}(t)$ as in (3.8) with $\alpha_{i j} \equiv 0$ and possible unbounded delays $\tau_{i j}^{k}(t)$. Linear systems where the terms with (either discrete or distributed) finite diagonal delays play an important role, and thus are separated from the terms with unbounded delay, will be analyzed in Section 4.

With this notation, in the sequel one or more of the assumptions below will be imposed:

(H3) there exist $\alpha_{0}>0$ and functions $\mu_{i j}:(-\infty, 0] \rightarrow[0, \infty)$ such that $\left|\nu_{i j}(t, s)\right| \leq\left|\mu_{i j}(s)\right|$ for all $t \geq 0, s \leq 0$ and $\int_{-\infty}^{0} \mathrm{e}^{-\alpha_{0} s} g(s) d\left|\mu_{i j}\right|(s)<\infty, i, j=1, \ldots, n ;$

(H4) there exist vectors $u, v>0$ and $T \geq 0$ such that $M(t) v \geq u$ for $t \geq T$;

(H5) there exist a vector $v>0, \alpha>1$ and $T \geq 0$ such that $\widehat{D}(t) v \geq \alpha A(t) v$ for $t \geq T$.

Some comments about these hypotheses follow. 
Remark 3.3. Observe that (H3) is trivially satisfied by linear equations with finite delay $\tau$, since one may take $\nu_{i j}(t, s) \equiv \nu_{i j}(t,-\tau)$ for $s \leq-\tau$. On the other hand, if $g(s)=\mathrm{e}^{-\gamma s}(s \leq 0)$ for some $\gamma>0$ and $\mathcal{C}=C_{\gamma}^{0}$ (or $\mathcal{C}=C_{\gamma}$ as defined in Section 2), it is clear that $C_{\gamma}^{0} \subset C_{\gamma_{1}}^{0}$ for any $\gamma_{1}>\gamma$. Therefore, in this case (H3) implies that the operators $L_{i j}(t) \in\left(C_{\gamma}^{0}(\mathbb{R})\right)^{\prime}$ are uniformly bounded for $t \geq 0$ by the operator $T_{i j}$ in $\left(C_{\gamma_{1}}^{0}(\mathbb{R})\right)^{\prime}$ (identified with the measure $\mu_{i j}$ ), for some $\gamma_{1}=\gamma+\alpha_{0}>\gamma$.

Remark 3.4. Both (H4) and (H5) are stronger versions of (H2), and they are equivalent under some boundedness conditions for the coefficients. In fact, if all the functions $a_{i j}(t)$ are bounded, then hypothesis (H4) implies (H5), since, with $v, u>0$ as in (H4), then (H5) holds with the same vector $v$ and any $1<\alpha<1+\min _{i}\left(u_{i} M_{i}^{-1}\right)$, where $0 \leq \sum_{j=1}^{n} a_{i j}(t) v_{j} \leq M_{i}$ in $[T, \infty)$. Similarly, if all the functions $(\widehat{D}(t) v)_{i}$ are bounded from below by a positive constant for $t>0$ large (which is clearly satisfied if (H5) holds and the functions $\sum_{j} a_{i j}(t)$ are all bounded from below by a positive constant), then hypothesis (H5) implies (H4): we have $(\widehat{D}(t) v)_{i}-\alpha\left(\sum_{j=1}^{n} a_{i j}(t) v_{j}\right) \geq 0$ and $(\widehat{D}(t) v)_{i} \geq m_{i}>0$, which implies (H4) with the same vector $v$ and $u=\left(1-\alpha^{-1}\right)\left(m_{1}, \ldots, m_{n}\right)$. In particular, if there are $m, M>0$ such that $m \leq \sum_{j=1}^{n} a_{i j}(t) \leq M$ for all $i$ and $t$ large, assumptions (H4) and (H5) are equivalent.

Lemma 3.3. For $\nu_{i j}$ continuous, with $\nu_{i j}(t, \cdot) \in M_{g}((-\infty, 0] ; \mathbb{R})$ and $\int_{-\infty}^{0} g(s) d_{s}\left|\nu_{i j}\right|(t, s)=1$, assume (H3). Then, for any $\eta>0$ there exists $\delta>0$ such that, for $i, j=1, \ldots, n$,

$$
\int_{-\infty}^{0} \mathrm{e}^{-\delta s} g(s) d_{s}\left|\nu_{i j}\right|(t, s)<1+\eta \text { for all } t \geq 0
$$

Proof. We prove (3.9) for each $i, j$ fixed. Define $F(t, \alpha)=\int_{-\infty}^{0} \mathrm{e}^{-\alpha s} g(s) d_{s}\left|\nu_{i j}\right|(t, s)$. By (H3), $F(t, \alpha)$ is well-defined for $(\alpha, t) \in\left[0, \alpha_{0}\right] \times[0, \infty)$ and

$$
\mathbf{F}(\alpha):=\sup _{t \geq 0} F(t, \alpha) \leq \int_{-\infty}^{0} \mathrm{e}^{-\alpha_{0} s} g(s) d\left|\mu_{i j}\right|(s)=: C<\infty, \quad \alpha \in\left[0, \alpha_{0}\right] .
$$

Moreover, $\alpha \mapsto F(t, \alpha)(t \geq 0)$ and $\mathbf{F}(\alpha)$ are non-decreasing in $\alpha \in\left[0, \alpha_{0}\right]$, with $F(t, 0)=\mathbf{F}(0)=1$.

If $C=1$, then $\mathbf{F} \equiv 1$ and (3.9) holds for all $\delta \in\left[0, \alpha_{0}\right]$. Otherwise, for any $\varepsilon>0$ given, choose $M>0$ such that

$$
\int_{-\infty}^{-M} \mathrm{e}^{-\alpha_{0} s} g(s) d\left|\mu_{i j}\right|(s)<\varepsilon / 3 .
$$

Since $f(s, \alpha):=\mathrm{e}^{-\alpha s}$ is uniformly continuous on $[-M, 0] \times\left[0, \alpha_{0}\right]$, there exists $\sigma>0$ such that $\left|\mathrm{e}^{-\alpha s}-\mathrm{e}^{-\beta s}\right|<\varepsilon / 3$ for any $(s, \alpha),(s, \beta) \in[-M, 0] \times\left[0, \alpha_{0}\right]$ with $|\alpha-\beta|<\sigma$. Thus for $t \geq 0$ and $\alpha, \beta \in\left[0, \alpha_{0}\right]$ with $|\alpha-\beta|<\sigma$,

$$
|F(t, \alpha)-F(t, \beta)| \leq 2 \int_{-\infty}^{-M} \mathrm{e}^{-\alpha_{0} s} g(s) d\left|\mu_{i j}\right|(s)+\int_{-M}^{0}\left|\mathrm{e}^{-\alpha s}-\mathrm{e}^{-\beta s}\right| g(s) d_{s}\left|\nu_{i j}\right|(t, s)<\varepsilon .
$$

This estimate and the monotonicity properties of $\mathbf{F}$ and $F(t, \cdot)$ imply that $|\mathbf{F}(\alpha)-\mathbf{F}(\beta)| \leq \varepsilon$ if $|\alpha-\beta|<\sigma$. This shows that $\mathbf{F}$ is continuous on $\left[0, \alpha_{0}\right]$. Therefore for any $\eta>0$ with $1+\eta \leq C$, there exists $\delta \in\left(0, \alpha_{0}\right)$ such that

$$
\int_{-\infty}^{0} \mathrm{e}^{-\delta s} g(s) d_{s}\left|\nu_{i j}\right|(t, s) \leq \mathbf{F}(\delta)=1+\eta, \quad t \geq 0 .
$$

The proof is complete. 
We are ready to state the main results of this section. We first address the exponential stability of (3.6). For $D(t)=\left[d_{i j}(t)\right]$ with $d_{i j}(t)$ continuous and bounded, it follows e.g. from [7, Proposition 6.3] that the ODE $x^{\prime}(t)=-D(t) x(t)$ is exponentially asymptotically stable if (H4) holds, i.e., if for $\widehat{D}(t)$ as in (3.2) there are positive vectors $v, u$ such that $\widehat{D}(t) v \geq u$ for $t \gg 1$. The generalization of this result to DDEs (3.6) is the subject of the next theorem, and does not require the a priori boundedness of all the coefficients.

Theorem 3.1. For system (3.6), assume (H1), (H3), and one of the following sets of conditions:

(i) (H4) is satisfied and $a_{i j}(t)$ are bounded functions on $\mathbb{R}^{+}$for all $i, j=1, \ldots, n$;

(ii) (H5) is satisfied and, for $v=\left(v_{1}, \ldots, v_{n}\right)>0$ as in (H5), $\liminf _{t \rightarrow \infty}\left(d_{i i}(t) v_{i}-\sum_{j \neq i}\left|d_{i j}(t)\right| v_{j}\right)>$ 0 for $i=1, \ldots, n$.

Then, (3.1) is exponentially asymptotically stable.

Proof. (i) Denote $d_{i}(t):=d_{i i}(t)$ and $a_{i j}(t)$ as in (3.5). After rescaling the variables we take $v=\mathbf{1}$ in (H4), and consider $T, m, M>0$ such that $d_{i}(t)-\sum_{j \neq i}\left|d_{i j}(t)\right|-\sum_{j} a_{i j}(t) \geq m$ and $\sum_{j} a_{i j}(t) \leq M$, for all $t \geq T, i=1, \ldots, n$. For $0<\eta<m(1+M)^{-1}$, we have

$$
d_{i}(t)-\eta-\sum_{j \neq i}\left|d_{i j}(t)\right|-(1+\eta) \sum_{j} a_{i j}(t)>0, \quad t \geq T, i=1, \ldots, n .
$$

Next, by Lemma 3.3, choose $\delta \in(0, \eta)$ such that (3.9) holds. With $e(t)=\delta$, the operators $\tilde{L}_{i j}(t)$ in (3.3), given by

$$
\tilde{L}_{i j}(t) \phi_{j}=L_{i j}(t)\left(\mathrm{e}^{-\delta \cdot} \phi_{j}\right)=a_{i j}(t) \int_{-\infty}^{0} \mathrm{e}^{-\delta s} \phi_{j}(s) d_{s} \nu_{i j}(t, s)
$$

for all $i, j$ and $t \geq 0, \phi=\left(\phi_{1}, \ldots, \phi_{n}\right) \in \mathcal{C}$, are well defined; moreover, $\left\|\tilde{L}_{i j}(t)\right\| \leq(1+\eta) a_{i j}(t)$. From Lemma 3.2, it follows that (3.1) is exponentially asymptotically stable.

(ii) As above, take $v=\mathbf{1}$ in (H5). Choose $m>0$ such that $d_{i}(t)-\sum_{j \neq i}\left|d_{i j}(t)\right| \geq m$ for $t \geq T, 1 \leq i \leq n$, and $\eta>0$ with $1+\eta<\alpha$. Next, choose $\delta>0$ such that (3.9) holds and $\delta<m\left[1-(1+\eta) \alpha^{-1}\right]$. We obtain

$$
\begin{aligned}
d_{i}(t)-\delta-\sum_{j \neq i}\left|d_{i j}(t)\right|-(1+\eta) \sum_{j} a_{i j}(t) & \geq\left[1-(1+\eta) \alpha^{-1}\right]\left(d_{i}(t)-\sum_{j \neq i}\left|d_{i j}(t)\right|\right)-\delta \\
& \geq m\left[1-(1+\eta) \alpha^{-1}\right]-\delta>0
\end{aligned}
$$

and again the result follows from Lemma 3.2 with $e(t)=\delta$.

A closer look to the proof of (i) above shows that, in the case of finite delays, the functions $a_{i j}(t)=\left\|L_{i j}(t)\right\|$ are not required to be bounded.

Corollary 3.1. Assume (H1), (H3), $\liminf _{t \rightarrow \infty} d_{i i}(t)>0$ and that there exist $v=\left(v_{1}, \ldots, v_{n}\right)>0$, $T \geq 0$ and $\alpha>1$ such that $d_{i i}(t) v_{i} \geq \alpha\left(\sum_{j}\left[\left(1-\delta_{i j}\right)\left|d_{i j}(t)\right|+a_{i j}(t)\right] v_{j}\right)$ for $t \geq T, i=1, \ldots, n$, where $\delta_{i j}=1$ if $i=j, \delta_{i j}=0$ if $i \neq j$. Then (3.6) is exponentially asymptotically stable.

Proof. This is a particular case of (ii), if in (3.6) we take $d_{i j}(t)=0$ and replace $L_{i j}(t) \psi$ by $d_{i j}(t) \psi(0)+$ $L_{i j}(t) \psi$, for all $j \neq i$. 
Consider now the case of autonomous linear DDEs of the form

$$
x_{i}^{\prime}(t)=-D x(t)+L x_{t}, \quad t \in I,
$$

where $D=\left[d_{i j}\right] \in \mathbb{R}^{n \times n}$ with $d_{i}:=d_{i i}>0$, and $L=\left(L_{1}, \ldots, L_{n}\right) \in L\left(\mathcal{C}, \mathbb{R}^{n}\right)$. Of course, for (3.12) the asymptotic and exponential stabilities coincide. As before, write the components $L_{i}$ of $L$ as $L_{i}(\phi)=\sum_{j=1}^{n} L_{i j}\left(\phi_{j}\right)$, define the $n \times n$ matrices

$$
D=\left[d_{i j}\right], \quad \widehat{D}=\left[\widehat{d}_{i j}\right], \quad A=\left[a_{i j}\right], \quad M=\widehat{D}-A,
$$

where $\widehat{d}_{i i}=d_{i i}, \widehat{d}_{i j}=-\left|d_{i j}\right|$ if $i \neq j$ and $a_{i j}=\left\|L_{i j}\right\|$, for $i, j=1, \ldots, n$. In this situation, condition (H2) simply says that $M v \geq 0$ for some positive vector $v$. From Lemma 3.1, it follows that (H2) implies that (3.12) is stable, thus all the roots of its characteristic equation have nonpositive real parts. It is easy to see that (H2) is not sufficient to guarantee that (3.12) is exponentially stable in any space $\mathcal{C}$ (see e.g. Example 5.4 in the last section). On the other hand, the next theorem asserts that, if 0 is not a characteristic value, then asymptotic stability follows with $M$ satisfying a property weaker than (H2). To show this, some algebraic definitions and properties are recalled below.

Definition 3.1. A square matrix $N=\left[n_{i j}\right]$ with nonpositive off-diagonal entries (i.e., $n_{i j} \leq 0$ for $i \neq j)$ is said to be an M-matrix, respectively a non-singular M-matrix, if all its eigenvalues have non-negative, respectively positive, real parts.

If $n_{i j} \leq 0$ for $i \neq j$, it is well-known that $N=\left[n_{i j}\right]$ is a non-singular M-matrix if and only if there exists a positive vector $v$ such that $N v>0$; and if there is a vector $v \geq 0$ such that $N v \geq 0$, then $N$ is an M-matrix; the converse is not always true (but it is valid in the case of irreducible matrices). See e.g. [6], also for further properties of these matrices.

Theorem 3.2. Consider in $\mathcal{C}$ the autonomous linear system $x^{\prime}(t)=-D x(t)+L x_{t}$, where $D=$ $\left[d_{i j}\right]_{n \times n}$ and $L=\left(L_{1}, \ldots, L_{n}\right) \in L\left(\mathcal{C} ; \mathbb{R}^{n}\right)$. For $L_{i}(\varphi)=\sum_{j} L_{i j}\left(\varphi_{j}\right)$ for $\varphi=\left(\varphi_{1}, \ldots, \varphi_{n}\right) \in \mathcal{C}$, define the matrices

$$
M_{0}=-D+\left[L_{i j}(1)\right], \quad M=\widehat{D}-A,
$$

where $D, A, M$ are as in (3.13). If $\operatorname{det} M_{0} \neq 0$ and $M$ is an $M$-matrix, then (3.12) is (exponentially) asymptotically stable.

This theorem generalizes the criterion obtained in [13, Theorems 2.3 and 2.6], where det $M_{0} \neq 0$ and $M$ an M-matrix were proven to be sharp conditions for the absolute exponential stability of autonomous linear DDEs with finite delays. Although we are considering infinite delays in $x^{\prime}(t)=$ $-D x(t)+L x_{t}$, the proof of Theorem 3.2 follows along arguments similar to the ones in [13], and is therefore omitted.

Remark 3.5. In [28], Ngoc and Cao investigated the exponential stability of a nonautonomous linear system in $C_{\gamma}$ (for some $\gamma>0$ ) written in the abstract form

$$
x^{\prime}(t)=-D_{0}(t) x(t)+\sum_{k=1}^{\infty} D_{k}(t) x\left(t-\tau_{k}(t)\right)+\int_{-\infty}^{0} B(t, s) x(t+s) d s,
$$

where the discrete delays $\tau_{k}(t) \geq 0$ are all uniformly bounded by positive constants $\tau_{k}$, the $n \times n$ matrices of functions $D_{k}(t)=\left[d_{i j}^{k}(t)\right], B(t, s)$ are all bounded by autonomous matrices $D_{k}, B(s)$ for 
all $k \in \mathbb{N}_{0}, t \geq 0, s \leq 0$, with the exception of the diagonal entries $d_{i i}^{0}(t)$ of $D_{0}(t)$, which are only required to be bounded from below by a positive constant $c_{i}>0, i=1, \ldots, n$. Note that (3.1) encompasses systems of the form (3.15). Ngoc and Cao [28, Theorem 3.3] derived the exponential stability of (3.15) in some space $C_{\gamma_{0}}$ with $\gamma_{0} \in(0, \gamma)$ under additional stronger conditions. Besides the uniform bounds of the entries of matrices $D_{k}(t), B(t, s)$ as described above, it was further imposed: a hypothesis with the role of the present assumption (H3), $\sum_{k=1}^{\infty} \mathrm{e}^{\gamma \tau_{k}}\left\|D_{k}\right\|<\infty$, and that there exists an $n \times n$ non-singular $M$-matrix of constants $M$ such that, with our notations, $M(t) \geq M$ for all $t \geq 0$. This latter requirement is stronger than either (H4) or (H5). More recently, Ngoc et al. 29] considered linear DDEs (3.1) but only with finite delay, without requiring a priori uniforms bounds of all the coefficients; for the situation of finite delay, Theorem 3.2.(iv) in [29] is exactly the criterion expressed in our Theorem 3.1.(i), nevertheless the other criteria in [29, Theorem 3.2.(i)-(iii)] are more restrictive than the ones in our Theorem 3.1, even for the situation of finite delay.

We now establish criteria for the asymptotic stability (but not necessarily exponential stability) of (3.6) without imposing (H3), nor that part of the coefficients are bounded. Therefore, we need to restrict the class (3.1), in order to guarantee the existence of some function $e(t)$ satisfying the assumptions in Lemma 3.2. Henceforth, in this section we treat linear equations (3.6) of the particular form

$$
x_{i}^{\prime}(t)=-\sum_{j=1}^{n} d_{i j}(t) x_{j}(t)+\sum_{j=1}^{n} a_{i j}(t) \int_{-\tau_{i j}(t)}^{0} x_{j}(t+s) d_{s} \nu_{i j}(t, s), i=1, \ldots, n, t \geq 0,
$$

for some nonnegative, continuous and possibly unbounded delay functions $\tau_{i j}(t)$. With the previous notation, we may suppose that $\nu_{i j}(t, \cdot):\left[-\tau_{i j}(t), 0\right] \rightarrow \mathbb{R}$ are bounded variation functions and satisfy

$$
\int_{-\tau_{i j}(t)}^{0} g(s) d_{s}\left|\nu_{i j}\right|(t, s)=1, \quad t \geq 0, i, j=1, \ldots, n
$$

Alternatively, we may still consider $\nu_{i j}(t, \cdot):(-\infty, 0] \rightarrow \mathbb{R}$ as in (3.5) and take $\nu_{i j}(t, s)=\nu_{i j}\left(t,-\tau_{i j}(t)\right)$ for $s<-\tau_{i j}(t)$ and $t \geq 0$. For (3.16), Lemma 3.2 gives the criterion below.

Theorem 3.3. Consider (3.16) with $\tau_{i j}:[0, \infty) \rightarrow[0, \infty)$ continuous, and $\operatorname{set} \tau(t)=\max _{1 \leq i, j \leq n} \tau_{i j}(t)$. Assume (H1), where now the bounded variation functions $\nu_{i j}(t, s)$ satisfy (3.17). In addition, suppose that $\tau(t) \leq t$ for $t \gg 1$ and that there exists a measurable, locally integrable function $e: \mathbb{R} \rightarrow \mathbb{R}^{+}$, a vector $v>0$ and $T \geq 0$, such that the following conditions are satisfied:

(i) $\left[\widehat{D}(t)-\mathrm{e}^{\int_{t-\tau(t)}^{t} e(u) d u} A(t)-e(t) I\right] v \geq 0$ for $t \geq T$, where $I$ is the $n \times n$ identity matrix;

(ii) $\int_{0}^{\infty} e(t) d t=\infty$.

Then (3.16) is asymptotically stable.

Proof. The operators in (3.3) are given by $\tilde{L}_{i j}(t) \varphi=a_{i j}(t) \int_{-\tau_{i j}(t)}^{0} \varphi(s) \mathrm{e}^{\int_{t+s}^{t} e(u) d u} d_{s} \nu_{i j}(t, s)$ (for $\left.\varphi \in C_{g}^{0}(\mathbb{R})\right)$, thus we have the estimates

$$
\tilde{a}_{i j}(t):=\left\|\tilde{L}_{i j}(t)\right\| \leq a_{i j}(t) \mathrm{e}^{\int_{t-\tau_{i j}(t)}^{t} e(u) d u}, \quad t \gg 1, i, j=1, \ldots, n .
$$


Remark 3.6. It is clear that in (i) the matrix $\mathrm{e}^{\int_{t-\tau(t)}^{t} e(u) d u} A(t)$ can be replaced by the matrix $\left[a_{i j}(t) \mathrm{e}^{\int_{t-\tau_{i j}(t)}^{t} e(u) d u}\right]$. Note also that condition (i) expresses a restriction on the size of the delay functions $\tau_{i j}(t)$, nevertheless the delays need not be bounded (see e.g. Example 5.2 in Section 5).

Theorem 3.4. Under the general notations in Theorem 3.3 with $\tau(t) \leq t$ for $t$ large, assume (H1), and (H5). For some $v>0$ as in (H5) and some $T \geq 0$, suppose also that there exists a measurable, locally integrable function $e: \mathbb{R} \rightarrow \mathbb{R}^{+}$such that:

(i) $\min _{1 \leq i \leq n}(M(t) v)_{i} \geq e(t), t \geq T$;

(ii) $\int_{0}^{\infty} \bar{e}(\bar{t}) d t=\infty$;

(iii) $\sup _{t \geq T} \int_{t-\tau(t)}^{t} e(u) d u<\infty$.

Then (3.16) is asymptotically stable.

Proof. If (H5) holds, take a vector $v=\left(v_{1}, \ldots, v_{n}\right)>0$, a constant $\alpha>1$ and $T \geq 0$ such that

$$
d_{i}(t) v_{i}-\sum_{j \neq i}\left|d_{i j}(t)\right| v_{j} \geq \alpha\left(\sum_{j} a_{i j}(t) v_{j}\right) \quad \text { for } \quad 1 \leq i \leq n, t \geq T .
$$

Hence $e_{i}(t):=d_{i}(t) v_{i}-\sum_{j \neq i}\left|d_{i j}(t)\right| v_{j}-\sum_{j} a_{i j}(t) v_{j} \geq\left(1-\alpha^{-1}\right)\left(d_{i}(t) v_{i}-\sum_{j \neq i}\left|d_{i j}(t)\right| v_{j}\right)$. Set $E(t)=\delta \int_{0}^{t} e(u) d u$, with $\delta>0$ sufficiently small. After a scaling, take $v=1$. From (i) and (3.18),

$$
\begin{gathered}
d_{i}(t)-\delta e(t)-\sum_{j \neq i}\left|d_{i j}(t)\right|-\sum_{j} \tilde{a}_{i j}(t) \geq d_{i}(t)-\sum_{j \neq i}\left|d_{i j}(t)\right|-\delta e_{i}(t)-\mathrm{e}^{\delta \int_{t-\tau(t)}^{t} e(u) d u} \sum_{j} a_{i j}(t) \\
\geq\left(1-\delta\left(1-\alpha^{-1}\right)-\alpha^{-1} \mathrm{e}^{\delta \int_{t-\tau(t)}^{t} e(u) d u}\right)\left(d_{i}(t)-\sum_{j \neq i}\left|d_{i j}(t)\right|\right), \quad t \geq T, i=1, \ldots, n .
\end{gathered}
$$

From (iii), $1-\delta\left(1-\alpha^{-1}\right)-\alpha^{-1} \mathrm{e}^{\delta \int_{t-\tau(t)}^{t} e(u) d u} \rightarrow 1-\alpha^{-1}>0$ as $\delta \rightarrow 0^{+}$, and the conclusion follows by the previous theorem.

Remark 3.7. If in each equation there is only a single discrete delay for each variable, so that (3.16) reads as

$$
x_{i}^{\prime}(t)=-\sum_{j=1}^{n} d_{i j}(t) x_{j}(t)+\sum_{j=1}^{n} b_{i j}(t) x_{j}\left(t-\tau_{i j}(t)\right), i=1, \ldots, n, t \geq 0,
$$

in (3.18) we have the identity $\tilde{a}_{i j}(t)=a_{i j}(t) \mathrm{e}^{\int_{t-\tau_{i j}(t)}^{t} e(u) d u}$, where $a_{i j}(t)=\left|b_{i j}(t)\right|$. Thus, in the above theorem if we do not require the function $e(t)$ to be nonnegative, as long as it satisfies (i)-(iii), we are still able to conclude that all solutions of (3.20) tend to zero at infinity. This result also applies to equations with multiple terms with discrete delays, whose coefficients are of the same sign, i.e., to equations

$$
x_{i}^{\prime}(t)=-\sum_{j=1}^{n} d_{i j}(t) x_{j}(t)+\sum_{j=1}^{n} \sum_{k=1}^{p} d_{i j}^{k}(t) x_{j}\left(t-\tau_{i j}^{k}(t)\right), i=1, \ldots, n, t \geq 0,
$$

with $\left|\sum_{k=1}^{p} d_{i j}^{k}(t)\right|=\sum_{k=1}^{p}\left|d_{i j}^{k}(t)\right|$ for all $i, j, k$ and $t \geq 0$ large. 
Other criteria can be derived in a similar way. For instance, it is easy to verify that (H5) can be eliminated in Theorem 3.4 if (i), (iii) are replaced by slightly stronger conditions, as follows: (i') $\left.\min _{1 \leq i \leq n}(M(t)] v\right)_{i}=: e_{i}(t)>0$ and $e_{i}(t) \geq e(t), t \geq T$; (iii') $\lim \sup _{t \rightarrow \infty}\left(\mathrm{e}^{\int_{t-\tau(t)}^{t} e(u) d u}-1\right) \frac{(\widehat{D}(t)] v)_{i}}{e_{i}(t)}<$ $\infty$ for $1 \leq i \leq n$.

The scalar case is addressed in the following corollary:

Corollary 3.2. Consider the scalar linear DDE

$$
x^{\prime}(t)=-d(t) x(t)+L_{0}(t) x_{t}, \quad t \geq 0
$$

with $d(t)>0$ continuous and $L_{0}(t) \varphi=\beta(t) \int_{-\tau(t)}^{0} \varphi(s) d_{s} \nu(t, s)$ for $(t, \varphi) \in[0, \infty) \times C_{g}^{0}(\mathbb{R})$, where $\tau(t) \geq 0, \nu(t, s)$ are continuous in $t, \nu(t, \cdot) \in M_{g}((-\infty, 0] ; \mathbb{R})$ with $\int_{-\tau(t)}^{0} g(s) d_{s}|\nu|(t, s)=1$ so that $\beta(t)=\left\|L_{0}(t)\right\|$ for $t \geq 0$, and $\tau(t) \leq t$ for $t \geq 0$ large. Assume that there exist $T>0$ and $a$ measurable, locally integrable function $e: \mathbb{R} \rightarrow \mathbb{R}^{+}$such that one of the following conditions holds:

(a) $d(t)-\beta(t) \sup _{t \geq T} \mathrm{e}^{\int_{t-\tau(t)}^{t} e(u) d u} \geq e(t)$ for $t \geq T$, with $\int^{\infty} e(t) d t=\infty$;

(b) (i) $d(t) \geq \alpha \beta(t)$ for some $\alpha>1$ and $t \geq T$;

(ii) $e(t)$ satisfies $e(t) \leq d(t)-\beta(t), \int^{\infty} e(t) d t=\infty$ and $\sup _{t \geq T} \int_{t-\tau(t)}^{t} e(u) d u<\infty$.

Then (3.22) is globally asymptotically stable. If in addition $d(t) \geq c$ for some constant $c>0$ and $t$ sufficiently large, then (3.22) is globally exponentially stable.

Remark 3.8. In a recent paper [17], Győri and Horváth studied Halanay-type nonautonomous delay differential inequalities of the form

$$
x^{\prime}(t) \leq-d(t) x(t)+\beta(t) \sup _{s \in[t-\tau(t), t]} x(s), \quad t \geq t_{0},
$$

and

$$
x^{\prime}(t) \leq-d(t) x(t)+\beta(t) x(t-\tau(t)), \quad t \geq t_{0},
$$

where $d, \beta:\left[t_{0}, \infty\right) \rightarrow \mathbb{R}^{+}$are locally integrable, $\tau:\left[t_{0}, \infty\right) \rightarrow \mathbb{R}^{+}$is measurable with $t_{0}-\tau_{0} \leq$ $t-\tau(t) \rightarrow \infty$ as $t \rightarrow \infty$, for some $\tau_{0} \geq 0$. In fact, as mentioned, this more general framework, of locally integrable coefficients $d(t), \beta(t)$ and a measurable delay $\tau(t)$, could have been considered here. By using a different approach, in [17] the authors presented a comprehensive, refined analysis of the global attractivity of the zero solution of (3.23) and (3.24) (although the attractivity in [17. only concerns the nonnegative solutions of such inequalities). The main tools employed in [17] are generalized Halanay-type inequalities and the so-called generalized characteristic equation, applied to the nonautonomous scalar differential equation

$$
x^{\prime}(t)=-d(t) x(t)+\beta(t) x(t-\tau(t)), \quad t \geq t_{0},
$$

given by $e(t)+\beta(t) \mathrm{e}^{\int_{t-\tau(t)}^{t} e(s) d s}=d(t)$, where $e(t)$ is a locally integrable function on $\left[t_{0}-\tau_{0}, \infty\right)$. As in the present paper, in [17] the coefficients $d(t), \beta(t)$ are not required to be bounded, a constraint often imposed in the literature. Among other results, in particular Györi and Horváth gave a sharp criterion [17, Theorem 2.8] as follows: every nonnegative solution of (3.24) tends to zero at 
infinity if and only if condition (a) in Corollary 3.2 is satisfied by some measurable, locally integrable function $e(t)$, which however is not required to be nonnegative (cf. Remark 3.7 above). Thus, the sufficient condition of Györi and Horváth's result is a simple consequence of our Corollary 3.2 with $L_{0}(t) x_{t}=\beta(t) x(t-\tau(t))$. In [17, Theorem 3.6], it is was also established that if there exists $\alpha>1$ such that $d(t) \geq \alpha \beta(t)$ (for $t \geq t_{0}$ ) and $\sup _{t \geq 0} \int_{\max \left(t-\tau(t), t_{0}\right)}^{t}(d(u)-\beta(u)) d u<\infty$, then the zero solution of (3.23) or (3.24) is globally attractive if and only if $\int_{t_{0}}^{\infty}(d(t)-\beta(t)) d t=\infty$. When applied to (3.25), these conditions read as (b) of Corollary 3.2 with the choice $e(t)=d(t)-\beta(t)$, thus, again, the criterion in [17, Theorem 3.6] is a particular case of Corollary 3.2. Note that, not only (3.22) is more general than the scalar equation with one single discrete delay, but also its nonnegative solutions satisfy (3.23) with $\beta(t)=\left\|L_{0}(t)\right\|$. For the $n$-dimensional case, whether the hypotheses in Theorems 3.3 and 3.4 are optimal or not is an interesting question deserving future investigations.

\section{Linear systems with pure diagonal delays}

This section is devoted to the study of linear equations (1.1) which do not necessarily have a dominant diagonal negative feedback term without delay in each equation. In these circumstances, we shall assume the existence of one or several terms with diagonal finite delays, which may be either discrete or distributed, but which nevertheless dominate the effect of both the off-diagonal terms and the diagonal terms with infinite delay. For DDEs with only finite discrete delays, see the recent paper [5] for further interesting results on exponential stability depending on all delays.

We go back to a general linear DDE (1.1) in $\mathcal{C}$, and suppose that the terms with diagonal finite delays are separated from the others:

$$
x_{i}^{\prime}(t)=-\sum_{k=1}^{p} d_{i i}^{k}(t) \ell_{i}^{k}(t) x_{i, t}+L_{i}(t) x_{t}, \quad i=1, \ldots, n, t \geq 0,
$$

for bounded linear functionals $\ell_{i}^{k}(t) \in\left(C\left(\left[-\tau_{i i}^{k}(t), 0\right] ; \mathbb{R}\right)\right)^{\prime}$ and $\tau_{i i}^{k}(t) \geq 0$ bounded delays, $d_{i i}^{k}: \mathbb{R}^{+} \rightarrow \mathbb{R}$ continuous, $k=1, \ldots, p$, and, as before, $L(t) \in L\left(\mathcal{C}, \mathbb{R}^{n}\right)$ is given in coordinates by $L_{i}(t) x_{t}=$ $\sum_{j=1}^{n} L_{i j}(t) x_{j, t}$, for $i=1, \ldots, n$ and $t \geq 0$. Note that nondelayed terms may be included in (4.1). An extra condition on the operators $\ell_{i}^{k}(t)$ will be imposed, but first further comments on the phase space $\mathcal{C}=C_{g}^{0}\left(\mathbb{R}^{n}\right)$ are given.

Since the delays $\tau_{i i}^{k}(t)$ are bounded, say $\tau_{i i}^{k}(t) \leq r$ for all $t \geq 0, i=1, \ldots, n, k=1, \ldots, p$, the norms $\|\cdot\|_{\infty}$ and $\|\cdot\|_{g}$ are equivalent in $C\left(\left[-\tau_{i i}^{k}(t), 0\right] ; \mathbb{R}^{n}\right)$. Thus the space $M_{g}\left(\left[-\tau_{i i}^{k}(t), 0\right] ; \mathbb{R}\right)$ coincides with the usual space $\left(C\left(\left[-\tau_{i i}^{k}(t), 0\right] ; \mathbb{R}\right)\right)^{\prime}=B V\left(\left[-\tau_{i i}^{k}(t), 0\right] ; \mathbb{R}\right)$. For convenience, we write

$$
\ell_{i}^{k}(t) \varphi=\int_{-\tau_{i i}^{k}(t)}^{0} \varphi(s) d_{s} \xi_{i i}^{k}(t, s), \quad t \geq 0, \varphi \in C_{g}(\mathbb{R})
$$

for $\xi_{i i}^{k}(t, \cdot) \in B V\left(\left[-\tau_{i i}^{k}(t), 0\right] ; \mathbb{R}\right)$. With (4.2) and the previous notation for $L(t)$, (4.1) is given by

$$
\begin{aligned}
x_{i}^{\prime}(t)= & -\sum_{k=1}^{p} d_{i i}^{k}(t) \int_{-\tau_{i i}^{k}(t)}^{0} x_{i}(t+s) d_{s} \xi_{i i}^{k}(t, s) \\
& +\sum_{j=1}^{n} a_{i j}(t) \int_{-\infty}^{0} x_{j}(t+s) d_{s} \nu_{i j}(t, s), \quad i=1, \ldots, n, t \geq 0,
\end{aligned}
$$


where $a_{i j}(t), \nu_{i j}(t, s)$ are as in (3.5). In the sequel, the following conditions are assumed:

$\left(\mathrm{H} 1^{*}\right) \quad$ (i) $\ell_{i}^{k}, L_{i j}: \mathbb{R}^{+} \rightarrow L\left(C_{g}^{0}(\mathbb{R}), \mathbb{R}\right)$ and $d_{i i}^{k}: \mathbb{R}^{+} \rightarrow \mathbb{R}$ are continuous for all $i, j, k$;

(ii) the operators $\ell_{i}^{k}(t)$ are given by (4.2), where $\tau_{i i}^{k}: \mathbb{R}^{+} \rightarrow[0, r]$ are continuous (for some $r>0)$, and $\xi_{i i}^{k}(t, s)$ are measurable, continuous on $t$, nondecreasing on $s$ and normalized so that

$$
\xi_{i i}^{k}(t, 0)-\xi_{i i}^{k}\left(t,-\tau_{i i}^{k}(t)\right)=1, \quad t \geq 0,1 \leq i \leq n, 1 \leq k \leq p ;
$$

(iii) $d_{i}(t):=\sum_{k=1}^{p} d_{i i}^{k}(t)>0$ for $t \geq 0$ and $i=1, \ldots, n$.

We give a few comments about this general hypothesis. Clearly, the condition that $\xi_{i i}^{k}(t, s)$ are nondecreasing in $s \in\left[-\tau_{i i}^{k}(t), 0\right]$ expresses that the functionals $\ell_{i}^{k}(t)$ are nonnegative functionals [30]. Observe also that from $\left(\mathrm{H} 1^{*}\right)(\mathrm{ii})$ we obtain

$$
\ell_{i}^{k}(t)(1)=\int_{-\tau_{i i}^{k}(t)}^{0} d_{s} \xi_{i i}^{k}(t, s)=1, \quad t \geq 0,1 \leq i \leq n, 1 \leq k \leq p
$$

nevertheless the norm $\left\|\ell_{i}^{k}(t)\right\|=\left\|\ell_{i}^{k}(t)\right\|_{g}$ is given by $\left\|\ell_{i}^{k}(t)\right\|=\int_{-\tau_{i i}^{k}(t)}^{0} g(s) d_{s} \xi_{i i}^{k}(t, s)$. Here, although $d_{i}(t)=\sum_{k} d_{i i}^{k}(t)$ is required to be positive, each function $d_{i i}^{k}(t)$ may be either positive or negative, or change sign on $\mathbb{R}^{+}$. The case of discrete delays is included above, i.e., one may have $\ell_{i}^{k}(t) \varphi=$ $\varphi\left(-\tau_{i i}^{k}(t)\right)$ for several or all $i, k$; moreover, with $\tau_{i i}^{k}(t) \equiv 0$, in this situation $\ell_{i}^{k}(t) \varphi=\varphi(0)$. Thus, the situation with a diagonal term without delay is included in the present form (4.3). Note also that the operators $L_{i j}(t)$ in (4.1) may incorporate several terms with bounded delays, either discrete or distributed, as in (3.7) or (3.8). In other words, this framework encompasses linear DDEs of the form

$$
\begin{aligned}
x_{i}^{\prime}(t)=\sum_{j=1}^{n} & {\left[-d_{i j}^{0}(t) x_{j}(t)-\sum_{k=1}^{p} d_{i j}^{k}(t) \int_{-\tau_{i j}^{k}(t)}^{0} x_{j}(t+s) d_{s} \xi_{i j}^{k}(t, s)\right.} \\
& \left.+\alpha_{i j}(t) \int_{-\infty}^{0} x_{j}(t+s) d_{s} \nu_{i j}(t, s)\right], \quad i=1, \ldots, n, t \geq 0 .
\end{aligned}
$$

However only the diagonal zero or bounded delays have a relevant role in the results below.

Define the $n \times n$ matrix-valued functions

$$
\begin{aligned}
D_{k}(t)=\operatorname{diag}\left(d_{11}^{k}(t), \ldots, d_{n n}^{k}(t)\right)(1 \leq k \leq p), & D(t)=\operatorname{diag}\left(d_{1}(t), \ldots, d_{n}(t)\right), \\
A(t)=\left[\left\|L_{i j}(t)\right\|\right], & C^{\tau}(t)=\operatorname{diag}\left(c_{1}^{\tau}(t), \ldots, c_{n}^{\tau}(t)\right),
\end{aligned}
$$

and

$$
M(t)=D(t)-C^{\tau}(t)-A(t), \quad t \geq 0
$$

where

$$
\begin{aligned}
& d_{i}(t)=\sum_{k=1}^{p} d_{i i}^{k}(t), \\
& c_{i}^{\tau}(t)=\sum_{k=1}^{p}\left|d_{i i}^{k}(t)\right| \int_{t-\tau_{i i}^{k}(t)}^{t}\left(\sum_{l=1}^{p}\left|d_{i i}^{l}(u)\right| g\left(-\tau_{i i}^{l}(u)\right)+\sum_{j=1}^{n}\left\|L_{i j}(u)\right\|\right) d u, \quad i=1, \ldots, n .
\end{aligned}
$$


As mentioned, the situation with $\ell_{i}^{k}(t) \varphi=\varphi(0)$ for some or all $i, k$ is included in (4.1). Clearly, for systems of the form (3.1) (that is, of the form (4.5) with $d_{i j}^{k} \equiv 0$ for all $i, j$ and $k=1, \ldots, p$ ), then $C^{\tau}(t) \equiv 0$ and the matrix $M(t)$ reduces to $M(t)=\widehat{D}(t)-A(t)$ as in (3.2).

We now extend Lemma 3.1 to equations of the form (4.1), but beforehand we remark that the weight function $g$ of the phase space $C_{g}^{0}$ satisfies a useful property: since $\lim _{u \rightarrow 0^{-}} \frac{g(s+u)}{g(s)}=1$ uniformly on $(-\infty, 0]$, it follows that the set $\left\{\frac{g(s-r)}{g(s)}: s \leq 0\right\}$ is bounded, for any $r>0$.

Lemma 4.1. Consider the system (4.1) in the phase space $\mathcal{C}$. If assumption (H1*) holds and the matrix $M(t)$ in (4.7) satisfies (H2), then there exist $m \geq 1, T_{1} \geq 0$ and a vector $v>0$ such that the solutions $x(t)$ of (4.1) satisfy $|x(t)|_{v^{-1}} \leq m\left\|x_{t_{0}}\right\|_{g, v^{-1}}$ for $t \geq t_{0} \geq T_{1}$. In particular, (4.1) is (uniformly) stable on $\left[T_{1}, \infty\right)$.

Proof. We adapt the proof of Lemma 3.1, stressing however that the treatment of the delays $\tau_{i i}^{k}(t) \in$ $[0, r]$ requires special care. Let $T, v$ be as in $(\mathrm{H} 2)$ and $T_{1}:=\max \{2 r, T\}$.

Define $m=\sup _{s \leq 0} \frac{g(s-r)}{g(s)}$. Clearly $m \geq 1$. After rescaling the variables by $\bar{x}_{i}(t)=v_{i}^{-1} x_{i}(t)(1 \leq$ $i \leq n$ ), assume (H2) with $v=\mathbf{1}$ and take the norm $|x|=\max _{1 \leq i \leq n}\left|x_{i}\right|$. Note that $t-\tau_{i i}^{k}(t) \geq 0$ for $t \geq r$ and all $i$. Fix $\phi \in \mathcal{C}, t_{0} \geq T_{1}$, consider the solution $x(t)=x\left(t, t_{0}, \phi\right)$ of (4.1) and $J=\left[t_{0}, t_{0}+a\right]$, for any $a>0$. We claim that

$$
|x(t)| \leq \ell:=m\left\|x_{t_{0}}\right\|_{g} \quad \text { for } \quad t \in J .
$$

If this assertion fails to be true, there exists $i \in\{1, \ldots, n\}$ and $t_{1} \in\left(t_{0}, t_{0}+a\right]$ such that $u_{i}:=\left|x_{i}\left(t_{1}\right)\right|=\left|x\left(t_{1}\right)\right|=\max _{t \in J}|x(t)|>\ell$. Arguing as in the proof of Lemma 3.1, we derive that $\left\|x_{t}\right\|_{g} \leq u_{i}$ for $t \in J$. Next, suppose that $x_{i}\left(t_{1}\right)>0$ (the case $x_{i}\left(t_{1}\right)<0$ is analogous). From (H1*), we obtain

$$
\begin{aligned}
x_{i}^{\prime}(t)+d_{i}(t) x_{i}(t) & =\sum_{k=1}^{p} d_{i i}^{k}(t)\left(x_{i}(t)-\int_{-\tau_{i i}^{k}(t)}^{0} x_{i}(t+s) d_{s} \xi_{i i}^{k}(t, s)\right)+\sum_{j=1}^{n} L_{i j}(t) x_{j, t} \\
& =\sum_{k=1}^{p} d_{i i}^{k}(t) \int_{-\tau_{i i}^{k}(t)}^{0}\left(x_{i}(t)-x_{i}(t+s)\right) d_{s} \xi_{i i}^{k}(t, s)+\sum_{j=1}^{n} L_{i j}(t) x_{j, t} \\
& \leq \sum_{k=1}^{p} d_{i i}^{k}(t) \int_{-\tau_{i i}^{k}(t)}^{0}\left(\int_{t+s}^{t} x_{i}^{\prime}(u) d u\right) d_{s} \xi_{i i}^{k}(t, s)+u_{i} \sum_{j=1}^{n}\left\|L_{i j}(t)\right\| \\
& =\sum_{k=1}^{p} d_{i i}^{k}(t) \int_{t-\tau_{i i}^{k}(t)}^{t} x_{i}^{\prime}(u)\left(\int_{-\tau_{i i}^{k}(t)}^{u-t} d_{s} \xi_{i i}^{k}(t, s)\right) d u+u_{i} \sum_{j=1}^{n}\left\|L_{i j}(t)\right\| \\
& \leq \sum_{k=1}^{p}\left|d_{i i}^{k}(t)\right| \int_{t-\tau_{i i}^{k}(t)}^{t}\left|x_{i}^{\prime}(u)\right| d u+u_{i} \sum_{j=1}^{n}\left\|L_{i j}(t)\right\| .
\end{aligned}
$$

Using (4.3), we get

$$
\int_{t-\tau_{i i}^{k}(t)}^{t}\left|x_{i}^{\prime}(u)\right| d u \leq \int_{t-\tau_{i i}^{k}(t)}^{t}\left(\sum_{l=1}^{p}\left|d_{i i}^{l}(u)\right| \int_{-\tau_{i i}^{l}(u)}^{0}\left|x_{i}(u+s)\right| d_{s} \xi_{i i}^{l}(u, s)+\sum_{j=1}^{n}\left|L_{i j}(u) x_{j, u}\right|\right) d u .
$$


Consider any $k \in\{1, \ldots, p\}, j \in\{1, \ldots, n\}$. For $t \in J$ and $u \in\left[t-\tau_{i i}^{k}(t), t\right] \subset[t-r, t]$, we now show that

$$
\left\|x_{j, u}\right\| \leq u_{i}
$$

We separate the cases $u \geq t_{0}$ and $u<t_{0}$.

If $u \in\left[t_{0}, t\right]$, for $s \leq 0$ we obtain $\frac{\left|x_{j}(u+s)\right|}{g(s)} \leq\left|x_{j}(u+s)\right| \leq u_{i}$ if $u+s \in\left[t_{0}, t\right]$, and $\frac{\left|x_{j}(u+s)\right|}{g(s)}=$ $\frac{\left|x_{j}\left(t_{0}+s_{1}\right)\right|}{g(s)} \leq \frac{\left|x_{j}\left(t_{0}+s_{1}\right)\right|}{g\left(s_{1}\right)} \leq\left\|x_{j, t_{0}}\right\|_{g}<u_{i}$ if $u+s \leq t_{0}$, where $s_{1}=u+s-t_{0}$ (note that $s \leq s_{1} \leq 0$ ).

If $u \in\left[t-\tau_{i i}^{k}(t), t_{0}\right]$, for $s \leq 0$ define $s_{1}=u+s-t_{0}, s_{2}=u+s-\left(t_{0}-r\right)$. If $t_{0}-r \leq u+s$, then $-r \leq s_{1} \leq 0$ and $\frac{\left|x_{j}(u+s)\right|}{g(s)}=\frac{\left|x_{j}\left(t_{0}+s_{1}\right)\right|}{g\left(s_{1}\right)} \frac{g\left(s_{1}\right)}{g(s)} \leq m \frac{\left|x_{j}\left(t_{0}+s_{1}\right)\right|}{g\left(s_{1}\right)} \leq m\left\|x_{j, t_{0}}\right\|_{g}<u_{i}$. If $u+s<t_{0}-r$ since $s \leq s_{2} \leq 0$, we have $\frac{\left|x_{j}(u+s)\right|}{g(s)}=\frac{\left|x_{j}\left(t_{0}-r+s_{2}\right)\right|}{g(s)} \leq \frac{\left|x_{j}\left(t_{0}-r+s_{2}\right)\right|}{g\left(s_{2}\right)} \leq\left\|x_{j, t_{0}-r}\right\|_{g}$. Since

$$
\left\|x_{t_{0}-r}\right\|_{g}=\sup _{s \leq 0} \frac{\left|x\left(t_{0}-r+s\right)\right|}{g(s)} \leq m\left\|x_{t_{0}}\right\|, \quad t \geq T_{1}
$$

again we conclude that $\frac{\left|x_{j}(u+s)\right|}{g(s)}<u_{i}$. This proves (4.11).

The above estimates also show that, for all $i, k$ and $t \in J, u \in\left[t-\tau_{i i}^{k}(t), t\right], s \leq 0$, we have $\left|x_{i}(u+s)\right| \leq g(s) u_{i}$. Thus, using again (4.4) and the fact that $g$ is nonincreasing, we obtain

$$
\int_{-\tau_{i i}^{l}(u)}^{0}\left|x_{i}(u+s)\right| d_{s} \xi_{i i}^{l}(u, s) \leq g\left(-\tau_{i i}^{l}(u)\right) u_{i}
$$

Inserting (4.11), (4.12) in (4.10) yields

$$
\int_{t-\tau_{i i}^{k}(t)}^{t}\left|x_{i}^{\prime}(u)\right| d u \leq u_{i} \int_{t-\tau_{i i}^{k}(t)}^{t}\left(\sum_{l=1}^{p} \mid d_{i i}^{l}(u) g\left(-\tau_{i i}^{l}(u)\right)+\sum_{j=1}^{n}\left\|L_{i j}(u)\right\|\right) d u .
$$

The above inequalities (4.9), (4.13) and (H2) lead to

$$
x_{i}^{\prime}(t)+d_{i}(t) x_{i}(t) \leq u_{i}\left(c_{i}^{\tau}(t)+\sum_{j=1}^{n}\left\|L_{i j}(t)\right\|\right) \leq u_{i} d_{i}(t), \quad t \in J,
$$

thus $x_{i}(t) \leq x_{i}\left(t_{0}\right) \mathrm{e}^{-\int_{0}^{t} d_{i}(s) d s}+u_{i}\left(1-\mathrm{e}^{-\int_{0}^{t} d_{i}(s) d s}\right)$ for $t \in J$. For $t=t_{1}$, we derive $x_{i}\left(t_{0}\right)-u_{i} \geq 0$, which contradicts the assumption $u_{i}>\ell$. Hence, (4.8) holds and the proof is complete.

In a similar way, the arguments presented in Lemma 3.2 can be pursued for systems (4.1), as follows. Let $e: \mathbb{R} \rightarrow \mathbb{R}^{+}$be a measurable, locally integrable function with $\int_{0}^{\infty} e(t) d t=\infty$, for which the operators given by (3.3) are well-defined, $i, j=1, \ldots, n$, and denote $E(t)=\int_{0}^{t} e(u) d u$. By the change of variables $y(t)=\mathrm{e}^{E(t)} x(t)$, the linear DDE (4.1) is transformed into

$$
y_{i}^{\prime}(t)=e(t) y_{i}(t)-\sum_{k=1}^{p} d_{i i}^{k}(t) \tilde{\ell}_{i}^{k}(t)\left(y_{i, t}\right)+\sum_{j} \tilde{L}_{i j}(t)\left(y_{j, t}\right), i=1, \ldots, n, t \geq 0,
$$


where $\tilde{\ell}_{i}^{k}(t)\left(x_{i, t}\right)=\int_{-\tau_{i i}^{k}(t)}^{0} \mathrm{e}^{\int_{t+s}^{t} e(u) d u} x_{i}(t+s) d_{s} \xi_{i i}^{k}(t, s)$ and $\tilde{L}_{i j}(t)$ are as in (3.3) . For $k=1, \ldots, p$, define $\tilde{d}_{i i}^{k}(t)=d_{i i}^{k}(t) \int_{-\tau_{i i}^{k}(t)}^{0} \mathrm{e}^{\int_{t+s}^{t} e(u) d u} d_{s} \xi_{i i}^{k}(t, s)$, so that $d_{i i}^{k}(t) \tilde{\ell}_{i}^{k}(t)(1)=\tilde{d}_{i i}^{k}(t)$; note that $\left|\tilde{d}_{i i}^{k}(t)\right| \leq$ $\mathrm{e}^{\int_{t-\tau_{i i}^{k}(t)}^{t} e(u) d u}\left|d_{i i}^{k}(t)\right|$. For this system, we consider

$$
\tilde{M}(t)=\tilde{D}(t)-\tilde{C}^{\tau}(t)-\tilde{A}(t), \quad t \geq 0,
$$

with the matrices $\tilde{D}(t), \tilde{C}^{\tau}(t), \tilde{A}(t)$ defined according to the notation in (4.6). We have $\tilde{D}(t)=$ $\sum_{k=0}^{p} \tilde{D}_{k}(t)$ with $\tilde{D}_{0}(t)=-e(t) I$ for $I$ the $n \times n$ identity matrix and $\tau_{i i}^{0}(t) \equiv 0$ and $\tilde{D}_{k}(t)=$ $\operatorname{diag}\left(\tilde{d}_{11}^{k}(t), \ldots, \tilde{d}_{n n}^{k}(t)\right)$ for $k=1, \ldots, p, \tilde{A}(t)=\left[\left\|\tilde{L}_{i j}(t)\right\|\right]$ and $\tilde{C}^{\tau}(t)=\operatorname{diag}\left(\tilde{c}_{1}^{\tau}(t), \ldots, \tilde{c}_{n}^{\tau}(t)\right)$ with $\tilde{c_{i}^{\tau}}(t) \leq \widetilde{\widetilde{c_{i}^{\tau}}}(t)$, for

$\widetilde{c_{i}^{\tau}}(t):=\sum_{k=1}^{p} \mathrm{e}^{\int_{t-\tau_{i i}^{k}(t)}^{t} e(u) d u}\left|d_{i i}^{k}(t)\right| \int_{t-\tau_{i i}^{k}(t)}^{t}\left(e(s)+\sum_{l=1}^{p} \mathrm{e}^{\int_{t-\tau_{i i}^{l}(t)}^{t} e(u) d u}\left|d_{i i}^{l}(s)\right| g\left(-\tau_{i i}^{l}(s)\right)+\sum_{j=1}^{n}\left\|\tilde{L}_{i j}(s)\right\|\right) d s$.

If the transformed matrix $\tilde{M}(t)$ satisfies (H2), from Lemma 4.1 and reasoning along the lines of the proof of Lemma 3.2, we deduce that the original system (4.1) is asymptotically stable. Moreover, if one can choose $e(t)=\delta$ for some $\delta>0$ and $\tilde{M}(t)$ still satisfies (H2), the stability is exponential.

In an analogous way, Theorems 3.1 can now be adapted to the present setting.

Theorem 4.1. Consider system (4.1) in C. Assume (H1*), (H3) and one of the following conditions:

(i) the functions $d_{i i}^{k}(t),\left\|L_{i j}(t)\right\|$ are all bounded on $\mathbb{R}^{+}, i, j=1, \ldots, n, k=1, \ldots, p$, and the matrix $M(t)$ in (4.7) satisfies ( $\left.\mathrm{H}_{4}\right)$;

(ii) $\liminf _{t \rightarrow \infty} d_{i}(t)>0$ for $i=1, \ldots, n$, and there exist $\alpha>1, T \geq 0$ and a vector $v>0$ such that $D(t) v \geq \alpha\left(C^{\tau}(t)+A(t)\right) v$ for $t \geq T$.

Then (4.1) is exponentially asymptotically stable.

Proof. (i) Take $v=\mathbf{1}$ in (H4), so that there exists $m>0$ such that $d_{i}(t)-c_{i}^{\tau}(t)-\sum_{j}\left\|L_{i j}(t)\right\| \geq m$, for $i=1, \ldots, n$ and $t \geq T$. Since all the coefficients are bounded, there exists $\eta>0$ such that, for $t$ sufficiently large,

$$
d_{i}(t)-\eta-(1+\eta)^{2}\left[c_{i}^{\tau}(t)+\sum_{j}\left\|L_{i j}(t)\right\|\right]>0, \quad i=1, \ldots, n .
$$

Fix $\eta>0$ as above. By Lemma 3.3, choose $\delta \in(0, \eta)$ such that (3.9) holds. From the computations above, after the change of variables $y(t)=\mathrm{e}^{\delta t} x(t)$, for $\tilde{M}(t)$ in (4.15) we have

$\tilde{c_{i}^{\tau}}(t) \leq \widetilde{c_{i}^{\tau}}(t):=\sum_{k=1}^{p} \mathrm{e}^{\delta \tau_{i i}^{k}(t)}\left|d_{i i}^{k}(t)\right| \int_{t-\tau_{i i}^{k}(t)}^{t}\left(\delta+\sum_{l=1}^{p} \mathrm{e}^{\delta \tau_{i i}^{l}(s)}\left|d_{i i}^{l}(s)\right| g\left(-\tau_{i i}^{l}(s)\right)+(1+\eta) \sum_{j=1}^{n}\left\|L_{i j}(s)\right\|\right) d s$.

Since $\tau_{i i}^{k}(t) \leq r$, we may suppose that $\delta>0$ is chosen so that $\delta\left(1+r \mathrm{e}^{\delta r} \sum_{k=1}^{p}\left|d_{i i}^{k}(t)\right|\right)<\eta$ and $\mathrm{e}^{\delta \tau_{i i}^{k}(t)} \leq \mathrm{e}^{\delta r}<1+\eta$ for all $i, k$ and $t \geq 0$. We deduce that $\tilde{\tilde{c}}_{i}^{\tau}(t) \leq(1+\eta)^{2} c_{i}^{\tau}(t)+\delta r \mathrm{e}^{\delta r} \sum_{k=1}^{p}\left|d_{i i}^{k}(t)\right|$. From (4.16), it follows that $(\tilde{M}(t) \mathbf{1})_{i}=d_{i}(t)-\delta-\tilde{c}_{i}^{\tau}(t)-\sum_{j=1}^{n}\left\|\tilde{L}_{i j}(t)\right\|>0$ for $t \geq T, i=1, \ldots, n$, so the matrix $\tilde{M}(t)$ satisfies $(\mathrm{H} 2)$. The conclusion follows. 
(ii) As above, take $v=\mathbf{1}$ in the inequality $D(t) v \geq \alpha\left(C^{\tau}(t)+A(t)\right) v$, and consider $m>0$ such that $d_{i}(t) \geq m$ for $t \geq T$ and $i=1, \ldots, n$. Take $\eta>0$ with $1+\eta<\alpha$, where $\alpha>1$ is such that

$$
d_{i}(t) \geq \alpha\left[c_{i}^{\tau}(t)+\sum_{j}\left\|L_{i j}(t)\right\|\right], \quad i=1, \ldots, n,
$$

and by Lemma 3.3, choose $\delta \in(0, \eta)$ such that (3.9) holds and

$$
\delta \mathrm{e}^{-\delta r} \leq \eta m, \quad \delta \leq m\left[1-\left(1+\eta^{3}\right) \alpha^{-1}\right], \quad \mathrm{e}^{\delta \tau_{i i}^{k}(t)} \leq \mathrm{e}^{\delta r}<1+\eta .
$$

Note also that $\sum_{k=1}^{p}\left|d_{i i}^{k}(t)\right| g\left(-\tau_{i i}^{l}(s)\right) \geq \sum_{k=1}^{p}\left|d_{i i}^{k}(t)\right| \geq d_{i}(t) \geq m$ for $t \geq T$. For $\tilde{\widetilde{c_{i}^{\tau}}}(t)$ as above, after the change of variables $y(t)=\mathrm{e}^{\delta t} x(t)$, for $\tilde{M}(t)$ in (4.15) we have $\tilde{c_{i}^{\tau}}(t) \leq \widetilde{c_{i}^{\tau}}(t)$ with

$$
\begin{aligned}
\widetilde{\widetilde{c}}(t): & \leq \mathrm{e}^{\delta r} \sum_{k=1}^{p}\left|d_{i i}^{k}(t)\right| \int_{t-\tau_{i i}^{k}(t)}^{t}\left(\delta+\mathrm{e}^{\delta r} \sum_{l=1}^{p}\left|d_{i i}^{l}(s)\right| g\left(-\tau_{i i}^{l}(s)\right)+(1+\eta) \sum_{j=1}^{n}\left\|L_{i j}(s)\right\|\right) d s \\
& \leq \mathrm{e}^{\delta r} \sum_{k=1}^{p}\left|d_{i i}^{k}(t)\right| \int_{t-\tau_{i i}^{k}(t)}^{t}\left((1+\eta) \mathrm{e}^{\delta r} \sum_{l=1}^{p}\left|d_{i i}^{l}(s)\right| g\left(-\tau_{i i}^{l}(s)\right)+(1+\eta) \sum_{j=1}^{n}\left\|L_{i j}(s)\right\|\right) d s \\
& \leq(1+\eta)^{3} c_{i}^{\tau}(t) .
\end{aligned}
$$

From (4.17) and the above choice of $\delta$, for $t \geq T, i=1, \ldots, n$ we deduce that

$$
\begin{aligned}
(\tilde{M}(t) \mathbf{1})_{i} & =d_{i}(t)-\delta-\tilde{c}_{i}^{\tau}(t)-\sum_{j=1}^{n}\left\|\tilde{L}_{i j}(t)\right\| \\
& \geq d_{i}(t)-\delta-(1+\eta)^{3} c_{i}^{\tau}(t)-(1+\eta) \sum_{j=1}^{n}\left\|L_{i j}(t)\right\| \\
& \geq d_{i}(t)\left[1-(1+\eta)^{3} \alpha^{-1}\right]-\delta \geq 0,
\end{aligned}
$$

thus the matrix $\tilde{M}(t)$ satisfies (H2).

Remark 4.1. Note that when all the functions $d_{i i}^{k}(t),\left\|L_{i j}(t)\right\|, \tau_{i i}^{k}(t)$ are bounded on $\mathbb{R}^{+}$and $M(t)$ in (4.7) satisfies (H4), then $D(t) v \geq \alpha\left(C^{\tau}(t)+A(t)\right) v$ for $t \geq T$ (cf. (H5) and Remark 3.4).

Corollary 4.1. Consider the scalar linear equation

$$
x^{\prime}(t)=-\sum_{k=1}^{p} d_{k}(t) \int_{-\tau_{k}(t)}^{0} x(t+s) d_{s} \xi_{k}(t, s)+L_{0}(t) x_{t}, \quad t \geq 0,
$$

where $\tau_{k}(t), d_{k}(t)$ are continuous, $\tau_{k}(t) \geq 0$ and bounded on $\mathbb{R}^{+}, L_{0}(t) \varphi=\beta(t) \int_{-\infty}^{0} \varphi(s) d_{s} \nu(t, s)$ for $(t, \varphi) \in[0, \infty) \times C_{g}^{0}(\mathbb{R})$, with $\beta(t)=\left\|L_{0}(t)\right\|, s \mapsto \nu(t, s) \in M_{g}((-\infty, 0] ; \mathbb{R})$ and $\nu(t, s)$ satisfying (H3), $s \mapsto \xi_{k}(t, s)$ are nondecreasing and such that $\xi_{k}(t, 0)-\xi_{k}\left(t,-\tau_{k}(t)\right)=1$, and $\xi_{k}(t, s), \nu(t, s)$ are continuous in $t$, for $t \in \mathbb{R}^{+}, 1 \leq k \leq p$. In addition, suppose that one of the following conditions holds: 
(i) $d_{k}(t), \beta(t)$ are bounded, on $\mathbb{R}^{+}, 1 \leq k \leq p$, and there exist $T>0, \varepsilon>0$ such that

$$
\sum_{k=1}^{p} d_{k}(t) \geq \varepsilon+\sum_{k=1}^{p}\left|d_{k}(t)\right| \int_{t-\tau_{k}(t)}^{t}\left(\sum_{l=1}^{p}\left|d_{l}(s)\right| g\left(-\tau_{l}(s)\right)+\beta(s)\right) d s+\beta(t), \quad t \geq T
$$

(ii) $\liminf _{t \rightarrow \infty} \sum_{k=1}^{p} d_{k}(t)>0$ and there are $\alpha>1$ and $T \geq 0$ such that

$$
\sum_{k=1}^{p} d_{k}(t) \geq \alpha\left[\sum_{k=1}^{p}\left|d_{k}(t)\right| \int_{t-\tau_{k}(t)}^{t}\left(\sum_{l=1}^{p}\left|d_{l}(s)\right| g\left(-\tau_{l}(s)\right)+\beta(s)\right) d s+\beta(t)\right], \quad t \geq T .
$$

Then (4.18) is exponentially asymptotically stable.

The case of linear DDEs with only bounded delays, either discrete or distributed, is now addressed.

Corollary 4.2. Consider the linear DDE in $C\left([-r, 0] ; \mathbb{R}^{n}\right)$

$$
x_{i}^{\prime}(t)=-\sum_{j=1}^{n} \sum_{k=1}^{p} d_{i j}^{k}(t) \int_{-\tau_{i j}^{k}(t)}^{0} x_{j}(t+s) d_{s} \xi_{i j}^{k}(t, s), \quad t \geq 0, i=1, \ldots, n,
$$

and assume that:

(h1) $d_{i j}^{k}: \mathbb{R}^{+} \rightarrow \mathbb{R}, \tau_{i j}^{k}: \mathbb{R}^{+} \rightarrow \mathbb{R}^{+}$are continuous, and $\xi_{i j}^{k}(t, s)$ are continuous on $t \in \mathbb{R}^{+}$, of bounded variation on $s \in\left[-\tau_{i j}^{k}(t), 0\right]$, with $\operatorname{Var}_{s \in\left[-\tau_{i j}^{k}(t), 0\right]} \xi_{i j}^{k}(t, s)=1$, for $i, j=1, \ldots, n, k=1, \ldots, p$;

(h2) $s \mapsto \xi_{i i}^{k}(t, s)$ is nondecreasing for $t \geq 0,1 \leq i \leq n, 1 \leq k \leq p$;

(h3) $d_{i}(t):=\sum_{k=1}^{p} d_{i i}^{k}(t)>0$ for $t \geq T_{0}$ and all $i, k$, for some $T_{0} \geq 0$;

(h4) $\tau_{i j}^{k}(t) \in[0, r]$ for $t \geq 0,1 \leq i, j \leq n, 1 \leq k \leq p$;

(h5) all functions $d_{i j}^{k}$ are bounded on $\mathbb{R}^{+}$.

Define the matrices

$$
\widehat{D}(t)=\left[\widehat{d}_{i j}(t)\right], \quad C^{\tau}(t)=\operatorname{diag}\left(c_{1}^{\tau}(t), \ldots, c_{n}^{\tau}(t)\right),
$$

where

$$
\widehat{d}_{i j}(t)=\left\{\begin{array}{cc}
-\sum_{k=1}^{p}\left|d_{i j}^{k}(t)\right|, & i \neq j \\
d_{i}(t), & i=j
\end{array} \quad, c_{i}^{\tau}(t)=\sum_{k=1}^{p}\left|d_{i i}^{k}(t)\right| \int_{t-\tau_{i i}^{k}(t)}^{t} \sum_{j=1}^{n} \sum_{l=1}^{p}\left|d_{i j}^{l}(u)\right| d u(1 \leq i \leq n) .\right.
$$

If $M(t):=\widehat{D}(t)-C^{\tau}(t)$ satisfies (H4), then (4.21) is exponentially asymptotically stable. In particular, under the above conditions, the system

$$
x_{i}^{\prime}(t)=-\sum_{j=1}^{n} \sum_{k=1}^{p} d_{i j}^{k}(t) x_{j}\left(t-\tau_{i j}^{k}(t)\right), \quad t \geq 0, i=1, \ldots, n,
$$

is exponentially asymptotically stable.

Remark 4.2. In a recent paper, with a technique which makes uses of Bohl-Perron theorem and matrix norms, Berezansky et al. 4] gave sufficient conditions for the exponential asymptotic stability for linear systems of the form (4.23), with bounded discrete delays and all coefficients essentially 
bounded (although the more general framework of measurable, locally integrable functions was considered). However, in [4] not only the analysis is restricted to the case of discrete delays, but the hypotheses are stronger then the requirements in Corollary [4.2, for (4.23), in [4, Theorem 5] it is assumed that: (i) $d_{i}(t)$ are bounded away below from 0, (ii) there exists an autonomous matrix $N$ such that the matrix $N(t):=\left[m_{i j}(t) / d_{i}(t)\right]$, where $M(t)=\left[m_{i j}(t)\right]=\widehat{D}(t)-C^{\tau}(t)$ for the matrices in (4.22), satisfies $N(t) \geq N$ for $t \geq 0$, and (iii) $I-N$ is a non-singular M-matrix. We emphasize that this latter condition is equivalent to saying that $v-N v \geq u$ for some positive vectors $u, v$, which is more restrictive than saying that $M(t)$ satisfies (H4). The approach in [4] was extended most recently by the same authors in [5], where new criteria for the exponential asymptotic stability of system (4.23) depending on all the delays $\tau_{i j}(t)$ were given. An interesting open question is how to generalize the results in [5] to DDEs with distributed, and possibly unbounded, delays.

Several versions of Theorem 3.4 can be stated for the present framework. To avoid repetitions and keep this manuscript in a reasonable size, in the formulation below we assume that all the diagonal delays are bounded and all diagonal coefficients $d_{i i}^{k}(t)$ are nonnegative.

Theorem 4.2. For (4.21), and with the notation in the above corollary, assume (h1),(h2) and (h3') all functions $d_{i i}^{k}$ are nonnegative on $\mathbb{R}^{+}$, with $d_{i}(t):=\sum_{k=1}^{p} d_{i i}^{k}(t)>0$; Let

(h4') $\tau_{i i}^{k}(t) \in[0, r]$ for $t \geq 0,1 \leq i \leq n, 1 \leq k \leq p$.

$$
M(t)=D(t)-\widehat{C}^{\tau}(t)-\widehat{A}^{\tau}(t)
$$

where $D(t)=\operatorname{diag}\left(d_{1}(t), \ldots, d_{n}(t)\right), \widehat{A}^{\tau}(t)=\left[\widehat{a}_{i j}^{\tau}(t)\right], \widehat{C}^{\tau}(t)=\operatorname{diag}\left(\widehat{c}_{1}^{\tau}(t), \ldots, \widehat{c}_{n}^{\tau}(t)\right)$, with, for $1 \leq$ $i, j \leq n$ and $1 \leq k \leq p$,

$$
\widehat{a}_{i j}^{\tau}(t)=\left\{\begin{array}{cc}
\sum_{k=1}^{p} g\left(-\tau_{i j}^{k}(s)\right)\left|d_{i j}^{k}(t)\right|, & i \neq j \\
0, & i=j
\end{array}, \widehat{c}_{i}^{\tau}(t)=\sum_{k=1}^{p} d_{i i}^{k}(t) \int_{t-\tau_{i i}^{k}(t)}^{t} \sum_{l=1}^{p} \sum_{j=1}^{n} g\left(-\tau_{i j}^{l}(u)\right)\left|d_{i j}^{l}(u)\right| d u .\right.
$$

Assume that there exist $\alpha>1, T \geq 0$ and a vector $v>0$ and a measurable, locally integrable function $e: \mathbb{R} \rightarrow \mathbb{R}^{+}$such that:

(i) $\left.\min _{1 \leq i \leq n}(M(t)] v\right)_{i} \geq e(t), t \geq T$;

(ii) $D(t) v \geq \alpha\left(\widehat{C}^{\tau}(t)+\widehat{A}^{\tau}(t)\right), t \geq T$;

(iii) $\int_{0}^{\infty} e(t) d t=\infty$;

(iv) $\sup _{t \geq T} \int_{t-\tau(t)}^{t} e(u) d u<\infty$, for $\tau(t)=\max \left\{\tau_{i j}^{k}(t): 1 \leq i, j \leq n, 1 \leq k \leq p\right\}$.

Then (4.21) is asymptotically stable.

Proof. With the notations in (4.6), since $L_{i i}(t) \equiv 0, a_{i j}(t):=\left\|L_{i j}(t)\right\| \leq \sum_{k=1}^{p} g\left(-\tau_{i j}^{k}(s)\right)\left|d_{i j}^{k}(t)\right|=$ $\widehat{a}_{i j}(t)$ for $i \neq j$ and

$$
c_{i}^{\tau}(t)=\sum_{k=1}^{p} d_{i i}^{k}(t) \int_{t-\tau_{i i}^{k}(t)}^{t}\left(\sum_{l} g\left(-\tau_{i i}^{l}(u)\right) d_{i i}^{l}(u)+\sum_{j \neq i}\left\|L_{i j}(u)\right\|\right) d u \leq \widehat{c}_{i}^{\tau}(t) \quad \text { for } \quad 1 \leq i \leq n .
$$

Thus we get $A(t) \leq \widehat{A}^{\tau}(t)$ and $C^{\tau}(t) \leq \widehat{C}^{\tau}(t)$. By the change of variables $y(t)=\mathrm{e}^{\delta E(t)} x(t)$ where $E(t)=\int_{0}^{t} e(u) d u$ and $0<\delta \ll 1$, system (4.21) is transformed into (4.14) with $e(t)$ replaced by $\delta e(t)$. Arguing as in Theorem 3.4. one can show that the matrix $\tilde{M}(t)$ in (4.15) satisfies (H2), and the conclusion comes from Lemma 4.1 applied to $y(t)$, since solutions of (4.21) then satisfty $x(t)=\mathrm{e}^{-\delta E(t)} y(t) \rightarrow 0$ as $t \rightarrow \infty$. Details are omitted. 


\section{$5 \quad$ Examples}

We now illustrate our results with some simple examples. The notation for $L_{i j}(t)$ in Sections 3 and 4 will be used.

Example 5.1. Consider a linear system of the form

$$
x_{i}^{\prime}(t)=-d_{i} t^{2} x_{i}(t)+\sum_{j=1}^{n} \gamma_{i j}(t) \int_{-\tau_{i j}(t)}^{0} K_{i j}(s) x_{j}(t+s) d s, \quad t \geq 0, i=1, \ldots, n,
$$

where $d_{i}$ are positive constants, $\gamma_{i j}(t), \tau_{i j}(t)$ are continuous and nonnegative, $K_{i j}$ are bounded and integrable on $\left[-\tau_{i j}(t), 0\right], 1 \leq i, j \leq n$. Write $\tilde{K}_{i j}(t, s)=K_{i j}(s) \chi_{\left[-\tau_{i j}(t), 0\right]}(s)$ for $t \geq 0, s \leq 0$. Take $\mathcal{C}=C_{g}^{0}$ for any function $g$ satisfying the properties in (g) and such that, for some $\alpha>0$ and all $i, j$, $\sup _{t \in \mathbb{R}^{+}} \int_{-\tau_{i j}(t)}^{0} \mathrm{e}^{-\alpha s} g(s) d s<\infty$. Thus, (H3) is satisfied with any $\alpha_{0} \in(0, \alpha)$. The linear operators $L_{i j}(t)$ have norm $a_{i j}(t)=\gamma_{i j}(t)\left\|g \tilde{K}_{i j}(t, \cdot)\right\|_{L^{1}}=\gamma_{i j}(t) \int_{-\tau_{i j}(t)}^{0} g(s)\left|K_{i j}(s)\right| d s$. If

$$
\gamma_{i j}(t)=o\left(t^{2}\right) \quad \text { as } \quad t \rightarrow \infty
$$

then (H5) holds, and Theorem 3.1(ii) implies that (5.1) is exponentially asymptotically stable.

Example 5.2. Consider a linear system of the form

$$
x_{i}^{\prime}(t)=-d_{i}(t) x_{i}(t)+\sum_{j=1}^{n} \gamma_{i j}(t) \int_{t / 2}^{t} K_{i j}(s) x_{j}(s) d s, \quad t \geq 0, i=1, \ldots, n,
$$

where $d_{i}, \gamma_{i j}: \mathbb{R}^{+} \rightarrow \mathbb{R}^{+}$are continuous, with $\gamma_{i j}(t)$ bounded, $d_{i}(t) \geq \frac{1}{t}, K_{i j}$ are continuous, integrable on $\mathbb{R}^{+}$and there is $\alpha>0$ such that $\int_{0}^{\infty} \mathrm{e}^{\alpha s}\left|K_{i j}(s)\right| d s<\infty, 1 \leq i, j \leq n$. This equation has the form (3.16) with unbounded delays $\tau_{i j}(t)=t / 2$ for all $i, j$. Consider the space $\mathcal{C}=C_{\gamma}^{0}$, for some $\gamma \in(0, \alpha)$.

With the previous notation in (3.2) $, M(t)=\operatorname{diag}\left(d_{1}(t), \ldots, d_{n}(t)\right)-\left[\left\|L_{i j}(t)\right\|\right], L_{i j}(t) \phi=$ $\gamma_{i j}(t) \int_{-t / 2}^{0} K_{i j}(t+s) \phi(s) d s$ for $\phi \in C_{\gamma}^{0}(\mathbb{R}), 1 \leq i, j \leq n$. For any $\delta>0$ and $T=T(\delta) \geq 0$ sufficiently large,

$$
\begin{aligned}
a_{i j}(t):=\left\|L_{i j}(t)\right\| & =\gamma_{i j}(t) \mathrm{e}^{\gamma t} \int_{t / 2}^{t} \mathrm{e}^{-\gamma u}\left|K_{i j}(u)\right| d u \\
& \leq \gamma_{i j}(t) \mathrm{e}^{(\gamma-\alpha) t / 2} \int_{t / 2}^{t} \mathrm{e}^{\alpha u}\left|K_{i j}(u)\right| d u \leq \frac{\delta}{t}, \quad t \geq T, 1 \leq i, j \leq n .
\end{aligned}
$$

This shows that one can choose $c \in(0,1)$ and $T>0$ such that

$$
c d_{i}(t) \geq \sum_{j=1}^{n} a_{i j}(t), \quad e_{i}(t):=d_{i}(t)-\sum_{j=1}^{n} a_{i j}(t) \geq \frac{c}{t}, \quad t \geq T .
$$

With $e(t)=\frac{c}{t}$ for $t \geq T$, we have $\int^{\infty} e(t) d t=\infty$ and $\int_{t / 2}^{t} e(s) d s=c \log 2$ for $t \geq T$. From Theorem 3.4, it follows that the zero solution of (5.2) is globally atractive. 
Example 5.3. Consider the linear system

$$
x_{i}^{\prime}(t)=-\sum_{j=1}^{n} d_{i j} t^{\alpha} x_{j}(t)+\sum_{j=1}^{n} b_{i j} t^{\alpha} \int_{-\tau_{i j}(t)}^{0} x_{j}(t+s) d s, \quad i=1, \ldots, n,
$$

where $\alpha>0, b_{i j}, d_{i j} \in \mathbb{R}$ with $d_{i}:=d_{i i}>0$ for all $i$, and the delays $\tau_{i j}(t)$ are continuous with $0 \leq \tau_{i j}(t) \leq r_{i j}$ for some constants $r_{i j}>0, i, j=1, \ldots, n$.

With the notation in (3.2) we have $d_{i j}(t)=d_{i j} t^{\alpha}, a_{i j}(t)=\left|b_{i j}\right| \tau_{i j}(t) t^{\alpha} \leq\left|b_{i j}\right| r_{i j} t^{\alpha}$. Define the $n \times n$ matrices $\widehat{D}=\operatorname{diag}\left(d_{1}, \ldots, d_{n}\right)-\left[\left(1-\delta_{i j}\right)\left|d_{i j}\right|\right],|B|=\left[\left|b_{i j}\right| r_{i j}\right]$, where $\delta_{i j}=1$ if $i=j$ and $\delta_{i j}=0$ if $i \neq j$, and assume that

$$
N:=\widehat{D}-|B|
$$

is a non-singular M-matrix. This is equivalent to saying that there exists a positive vector $v$ such that $u:=N v>0$, hence (H5) is satisfied. From Theorem 3.1(ii) we deduce that (5.3) is exponentially asymptotically stable. Note however that none of coefficients is uniformly bounded on $\mathbb{R}^{+}$.

Example 5.4. Take a function $g$ satisfying $(\mathrm{g})$. Clearly $\psi \equiv 1 \in C_{g}^{0}(\mathbb{R})$ and $\|1\|_{g}=1$. By the HahnBanach theorem, there exists a functional $T \in\left(C_{g}^{0}(\mathbb{R})\right)^{\prime}$ such that $T(1)=\|T\|=1$. In $\mathcal{C}=C_{g}^{0}\left(\mathbb{R}^{2}\right)$, consider the planar system

$$
\begin{aligned}
& x_{1}^{\prime}(t)=-2 x_{1}(t)+T\left(x_{1, t}\right)+(-1)^{n} T\left(x_{2, t}\right) \\
& x_{1}^{\prime}(t)=-2 x_{2}(t)+T\left(x_{1, t}\right)+T\left(x_{2, t}\right)
\end{aligned}
$$

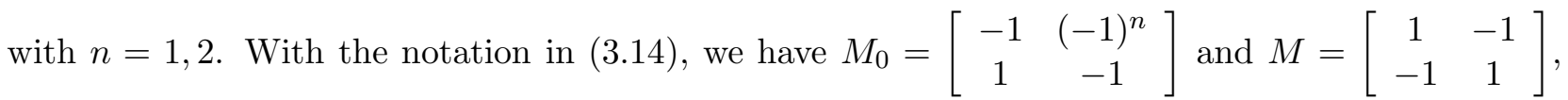
thus (H2) is satisfied with $v=(1,1)$. For $n=2, \lambda=0$ is a root of its characteristic equation, which is given by $\left(\lambda+2-T\left(\mathrm{e}^{\lambda \cdot}\right)\right)^{2}-T\left(\mathrm{e}^{\lambda \cdot}\right)^{2}=0$; since $(c, c)$ are equilibria for any $c$ constant, the system is not asymptotically stable. For the case $n=1$, $\operatorname{det} M_{0} \neq 0$ and from Theorem 3.3 the system is asymptotically stable.

Example 5.5. Consider the linear planar system

$$
\begin{aligned}
& x_{1}^{\prime}(t)=-\left(1+\cos ^{2} t\right) x_{1}\left(t-\tau_{11}(t)\right)+c_{1}\left(1+\sin ^{2} t\right) x_{2}\left(t-\tau_{12}(t)\right) \\
& x_{2}^{\prime}(t)=-\left(1+\sin ^{2} t\right) x_{2}\left(t-\tau_{22}(t)\right)+c_{2}\left(1+\cos ^{2} t\right) x_{1}\left(t-\tau_{21}(t)\right)
\end{aligned}
$$

where $c_{i} \neq 0$ and the delays $\tau_{i j}(t)$ are continuous and nonnegative on $\mathbb{R}^{+}$(and possibly unbounded), $i, j=1,2$.

First, consider the case $\tau_{11}(t)=\tau_{22}(t) \equiv 0$. With $\gamma_{i}=\left|c_{i}\right|, i=1,2$, and the notation in (3.2), we have $D(t)=\operatorname{diag}\left(1+\cos ^{2} t, 1+\sin ^{2} t\right)$ and

$$
M(t)=D(t)-A(t)=\left[\begin{array}{cc}
1+\cos ^{2} t & -\gamma_{1}\left(1+\sin ^{2} t\right) \\
-\gamma_{2}\left(1+\cos ^{2} t\right) & 1+\sin ^{2} t
\end{array}\right]
$$

For a vector $v=\left(1, v_{2}\right)$ with $v_{2}>0$, write $M(t) v=\left[\begin{array}{c}e_{1}(t) \\ e_{2}(t) v_{2}\end{array}\right]$. Since min $e_{1}(t)=1-2 v_{2} \gamma_{1}, \min e_{2}(t)=$ $1-2 v_{2}^{-1} \gamma_{2}$, if $4 \gamma_{1} \gamma_{2}<1$, i.e., if

$$
4\left|c_{1} c_{2}\right|<1,
$$


choosing $v_{2}$ such that $2 \gamma_{2}<v_{2}<\left(2 \gamma_{1}\right)^{-1}$, condition (H4) is satisfied with $v=\left(1, v_{2}\right)$. From Theorem [3.1. (5.4) is exponentially asymptotically stable.

Secondly, let $\tau_{11}(t)>0, \tau_{22}(t)>0$ for some $t>0$, but assume that all the delays $\tau_{i j}(t)$ are uniformly bounded, $\tau_{i j}(t) \leq r_{i j}$ on $\mathbb{R}^{+}$with $\max _{i, j=1,2} r_{i j}=r>0$, so that we work on $\mathcal{C}=$ $C\left([-r, 0] ; \mathbb{R}^{2}\right)$ (and $g \equiv 1$ on $\left.[-r, 0]\right)$. For $C^{\tau}(t)=\operatorname{diag}\left(c_{1}^{\tau}(t), c_{2}^{\tau}(t)\right)$ defined by (4.6), we have

$$
\begin{aligned}
c_{1}^{\tau}(t) & =\left(1+\cos ^{2} t\right) \int_{t-\tau_{11}(t)}^{t}\left(1+\cos ^{2} u+\gamma_{1}\left(1+\sin ^{2} u\right)\right) d u \\
c_{2}^{\tau}(t) & =\left(1+\sin ^{2} t\right) \int_{t-\tau_{22}(t)}^{t}\left(\gamma_{2}\left(1+\cos ^{2} u\right)+1+\sin ^{2} u\right) d u,
\end{aligned}
$$

and rough estimates give

$$
\begin{aligned}
& c_{1}^{\tau}(t) \leq 2 r_{11}\left(1+\cos ^{2} t\right)\left(1+\gamma_{1}\right), \\
& c_{2}^{\sigma}(t) \leq 2 r_{22}\left(1+\sin ^{2} t\right)\left(1+\gamma_{2}\right) .
\end{aligned}
$$

For $\widehat{D}(t), C^{\tau}(t)$ defined by (4.22), the matrix $\widehat{D}(t)$ coincides with the matrix $D(t)-A(t)$ in (5.5) . Proceeding as above we deduce that it is possible to choose a vector $v=\left(1, v_{2}\right)$ with $v_{2}>0$ such that $M(t)=\widehat{D}(t)-C^{\tau}(t)$ satisfies $(\mathrm{H} 4)$, provided that

$$
4\left|c_{1} c_{2}\right|<\left(1-2 r_{11}\left(1+\left|c_{1}\right|\right)\right)\left(1-2 r_{22}\left(1+\left|c_{2}\right|\right)\right),
$$

and from Corollary 4.2 we conclude that (5.4) is exponentially asymptotically stable. Note that when $r_{i i}=0(i=1,2)$, condition (5.7) reduces to (5.6).

Example 5.6. Consider the following scalar equation:

$$
x^{\prime}(t)=-\left(1+\cos ^{2} t\right) x\left(t-\tau_{1}(t)\right)+\left(1-\sin ^{2} t\right) x\left(t-\tau_{2}(t)\right)+b \int_{t / 2}^{t} \mathrm{e}^{-\gamma s} x(s) d s, \quad t \geq 0,
$$

where $b, \gamma>0, \tau_{i}: \mathbb{R}^{+} \rightarrow \mathbb{R}^{+}$are continuous with $\tau_{i}(t) \leq r_{i}, i=1,2$. With the notations in Corollary 4.1, $d(t)=1+\cos ^{2} t-\left(1-\sin ^{2} t\right)=1,\left|d_{1}(t)\right|+\left|d_{2}(t)\right|=2+\cos ^{2} t-\sin ^{2} t$ and $L_{0}(t)$ is the operator defined by $L_{0}(t) \phi=b \mathrm{e}^{-\gamma t} \int_{-t / 2}^{0} \mathrm{e}^{-\gamma s} \phi(s) d s$. Fix the phase space $\mathcal{C}=C_{\gamma}^{0}(\mathbb{R})$. Thus, the $\operatorname{norm} \beta(t):=\left\|L_{0}(t)\right\|$ is given by $\beta(t)=b \mathrm{e}^{-\gamma t} \int_{-t / 2}^{0} \mathrm{e}^{-2 \gamma s} d s=\frac{b}{2 \gamma}\left(1-\mathrm{e}^{-\gamma t}\right)$. With $r=\max \left(r_{1}, r_{2}\right)$, the coefficient $c^{\tau}(t)$ in (4.6) satisfies

$$
\begin{aligned}
c^{\tau}(t) & \leq\left(2+\cos ^{2} t-\sin ^{2} t\right) \int_{t-r}^{t}\left(\mathrm{e}^{\gamma r}\left(2+\cos ^{2} u-\sin ^{2} u\right)+\frac{b}{2 \gamma}\left(1-\mathrm{e}^{-\gamma u}\right)\right) d u \\
& \leq 3 r\left[3 \mathrm{e}^{\gamma r}+\frac{b}{2 \gamma}\right]+O\left(\mathrm{e}^{-\gamma t}\right), \quad \text { as } \quad t \rightarrow \infty .
\end{aligned}
$$

Thus, if $9 \mathrm{e}^{\gamma r} r+\frac{3 b}{2 \gamma}(1+r)<1$, then for any $\varepsilon>0$ there is $T>0$ such that

$$
c^{\tau}(t)+\beta(t) \leq 3\left[3 \mathrm{e}^{\gamma r} r+\frac{b}{2 \gamma}(1+r)\right]+\varepsilon<1, \quad t \geq T .
$$

In particular, (5.8) is exponentially asymptotically stable provided that $3 b<2 \gamma$ and $r>0$ is sufficiently small. 


\section{Acknowledgements}

This work was supported by National Funding from FCT - Fundação para a Ciência e a Tecnologia (Portugal) under project UIDB/04561/2020.

\section{References}

[1] Arino, O., Gőri, I., Pituk, M. (1996). Asymptotically diagonal delay differential systems. J. Math. Anal. Appl. 204, 701-728.

[2] Berezansky, L. Braverman, E. (2011). New stability conditions for linear differential equations with several delays. Abstract Appl. Anal. ID 1785668, 1-19.

[3] Berezansky, L. Braverman, E. (2020). Solution estimates for linear differential equations with delay. Appl. Math. Comput. 372. https://doi.org/10.1016/j.amc.2019.124962

[4] Berezansky, L. Diblík, J., Svoboda, Z., Smarda, Z. (2018). Exponential stability of linear delayed differential systems. Appl. Math. Comput. 320, 474-484.

[5] Berezansky, L. Diblík, J., Svoboda, Z., Smarda, Z. (2019). Exponential stability tests for linear delayed differential systems depending on all delays, J. Dyn. Diff. Equat. 31, 2095-2108.

[6] Berman, A., Plemmons, R. (1979). Nonnegative Matrices in the Mathematical Sciences, Academic Press, New York.

[7] Coppel,W.A. (1978). Dichotomies in Stability Theory, Lecture Notes in Mathematics, Vol. 629, Springer, Berlin.

[8] Diekmann, O., Gyllemberg, M. (2012). Equations with infinite delay: blending the abstract and the concrete. J. Differential Equations 252, 819-851.

[9] Driver, R.D. (1976). Linear differential systems with small delays. J. Differential Equations 21, 148-166.

[10] Faria, T. (2010). Stability and extinction for Lotka-Volterra systems with infinite delay. J. Dyn. Diff. Equat. 22, 299-324.

[11] Faria, T., Huang, W. (2005). Special solutions for linear functional differential equations and asymptotic behaviour. Differential Integral Equations 18, 337-360.

[12] Faria, T., Obaya, R., Sanz, A.M. (2018). Asymptotic behaviour for a class of non-monotone delay differential systems with applications. J. Dyn. Diff. Equat. 30, 911-935.

[13] Faria, T., Oliveira, J.J. (2008) Local and global stability for Lotka-Volterra systems with distributed delays and instantaneous feedbacks. J. Differential Equations 244, 1049-1079.

[14] Fehér, A., Márton, L., Pituk, M. (2019). Approximation of a linear autonomous differential equation with small delay, Simmetry 11, 1299.

[15] Garab, A., Pituk, M., Stavroulakis, I.P. (2019). A sharp oscillation criterion for a linear delay differential equation. Appl. Math. Lett. 93 (2019), 58-65.

[16] Gopalsamy, K. (1992). Stability and Oscillation in Delay Differential Equations of Population Dynamics, Kluwer Academic, Dordrecht. 
[17] Györi, I., Horváth, L. (2017). Sharp estimation for the solutions of delay differential and Halanay type inequalities. Discrete Contin. Dyn. Syst. Ser. A 37, 3211-3242.

[18] Györi, I., Horváth, L. (2018). Sharp estimation for the solutions of inhomogeneous delay differential and Halanay type inequalities. Electron. J. Qual. Theory Differ. Equ. 2018, Paper No. 54, 1-18.

[19] Haddock, J.R., Kuang, Y. (1992). Asymptotic theory for a class of nonautonomous delay differential equations. J. Math. Anal. Appl. 168, 147-162.

[20] Hale, J.K., Verduyn Lunel, S. M. (1993). Introduction to Functional Differential Equations, SpringerVerlag, New-York.

[21] Hatvani, L. (2016). Asymptotic stability of nonautonomous functional differential equations with distributed delays, Electron. J. Differential Equations 2016, Paper No. 302, 1-16.

[22] Hino, Y., Murakami, S., Naito, T. (1991). Functional Differential Equations with Infinite Delay, Springer-Verlag, New-York.

[23] Hofbauer, J., So, J.W.-H. (2000). Diagonal dominance and harmless off-diagonal delays. Proc. Amer. Math. Soc. 128, 2675-2682.

[24] Johnson, R.A., Sell, G.R. (1981). Smoothness of spectral subbundles and reducibility of quasiperiodic linear differential systems. J. Differential Equations 41, 262-288.

[25] Krisztin, T. (1991). On stability properties for one-dimensional functional-differential equations. Funkcial. Ekvac. 34, 241-256.

[26] Kuang, Y. (1993). Delay Differential Equations with Applications in Population Dynamics, Academic Press, New York.

[27] Murakami, S., Naito, T. (1989). Fading memory spaces and stability properties for functional differential equations with infinite delay. Funkcial. Ekvac. 32, 91-105.

[28] Ngoc, P.H.A., Tinh, C.T. (2016). Explicit criteria for exponential stability of time-varying systems with infinite delay, Math. Control Signals Systems 28, no. 1, Art. 4, 30 pp.

[29] Ngoc, P.H.A., Tran, T.B., Tinh, C.T, Huy, N.D. (2019). Novel criteria for exponential stability of linear nonautonomous functional differential equations. J. Syst. Sci. Complex. 32, no. 2, 479-495.

[30] Rudin, W. (1987). Real and Complex Analysis, 3rd. ed., McGraw-Hill Book Co., New York.

[31] Sacker, R.J., Sell, G.R. (1978). A spectral theory for linear differential systems. J. Differential Equations $27,320-358$.

[32] So, J.W.-H., Yu, J. S., Chen, M.-P., Asymptotic stability for scalar delay differential equations. Funkcial. Ekvac. 39 (1996), no. 1, 1-17.

[33] So, J. W.-H., Tang, X. H., Zou, X. (2004). Global attractivity for nonautonomous linear delay systems. Funkcial. Ekvac. 4, 25-40.

[34] Yoneyama, T. (1992). The 3/2 stability theorem for one-dimensional delay-differential equation with unbounded delay. J. Math. Anal. Appl. 165, 133-143. 